\title{
Gabbro, plagiogranite and associated dykes in the supra-subduction zone Evros Ophiolites, NE Greece
}

\author{
NIKOLAY BONEV* \& GÉRARD STAMPFLI $\dagger$
}

*Department of Geology and Paleontology, Sofia University 'St. Kliment Ohridski', BG-1504 Sofia, Bulgaria

$\dagger$ Institute of Geology and Paleontology, University of Lausanne, Anthropole, CH-1015 Lausanne, Switzerland

(Received 10 September 2007; accepted 7 January 2008; First published online 30 July 2008)

\begin{abstract}
The incomplete Evros ophiolites in NE Greece form a NE-SW-oriented discontinuous belt in the Alpine orogen of the north Aegean. Field data, petrology and geochemistry are presented here for the intrusive section and associated mafic dykes of these ophiolites. Bodies of high-level isotropic gabbro and plagiogranite in the ophiolite suite are cross-cut by NE-SW-trending boninitic and tholeiitic-boninitic affinity dykes, respectively. The dykes fill tensile fractures or faults, which implies dyke emplacement in an extensional tectonic regime. The tholeiitic-transitional boninitic gabbro is REE- and HFS-depleted relative to N-MORB, indicating derivation from melting of a refractory mantle peridotite source. Associated boninitic dykes are slightly LREE-enriched, showing mineral and wholerock geochemistry similar to the gabbro. The plagiogranite is a strongly REE-enriched high-silica trondhjemite, with textures and composition typical for an oceanic crust differentiate. Plagiogranitehosted tholeiitic and transitional boninitic dykes are variably REE-enriched. Geochemical modelling indicates origin of the plagiogranite by up to $75 \%$ fractional crystallization of basaltic magma similar to that producing the associated tholeiitic dykes. All mafic rocks have high LILE/HFSE ratios and negative $\mathrm{Ta}-\mathrm{Nb}-\mathrm{Ti}$ and $\mathrm{Ce}$ anomalies, typical for subduction zone-related settings. The mafic rocks show a similar trace-element character to the mafic lavas of an extrusive section in Bulgaria, suggesting they both form genetically related intrusive and extrusive suites of the Evros ophiolites. The field occurrence, the structural context, the petrology and geochemical signature of the studied magmatic assemblage provide evidence for its origin in a proto-arc (fore-arc) tectonic setting, thus tracing the early stages of the tectono-magmatic evolution of Jurassic arc-marginal basin system that has generated the supra-subduction type Evros ophiolites.
\end{abstract}

Keywords: isotropic gabbro, plagiogranite, tholeiitic-boninitic dykes, Evros ophiolites, Greece.

\section{Introduction}

The Mesozoic ophiolites in the Aegean domain of the eastern Mediterranean region (see Robertson, 2002 for review) represent fossil fragments of the Tethyan oceanic lithosphere within the Alpine collisional system (Fig. 1, inset; e.g. Robertson et al. 1996; Stampfli, 2000; Stampfli \& Borel, 2002, 2004). In the Dinaric-Hellenic Belt, these ophiolites present complex origins involving oceanic ridge and suprasubduction zone settings (Bortolotti et al. 2002; Pamic, Tomljenović \& Balen, 2002; Beccaluva et al. 2004; Bortolotti \& Principi, 2005; Dilek, Furnes \& Shallo, 2007).

The Jurassic ophiolites and associated sedimentary successions in Bulgaria and Greece comprise a lowgrade to non-metamorphic Mesozoic unit (Fig. 1), forming part of the ophiolitic belts in the Aegean domain. Despite broad temporal analogues to other ophiolites in this domain, these Jurassic ophiolites still remain unknown in terms of petrogenesis and tectono-magmatic evolution, and are thus critical for the geodynamics of Tethyan oceanic crust generation in the Eastern Mediterranean region. In Thrace, the ultramafic-mafic rocks in the low-grade unit were

*Author for correspondence: niki@gea.uni-sofia.bg designated as the Evros ophiolites (Magganas, Sideris \& Kokkinakis, 1991). Petrological studies on an extrusive sequence of these ophiolites have shown their supra-subduction zone origin to be in a marginal basinvolcanic arc setting (Magganas, Sideris \& Kokkinakis, 1991; Magganas, 2002). Similarly, the petrology of analogous arc-related extrusive rocks in the Eastern Bulgarian Rhodope further supported their origin and tectono-magmatic setting (Bonev \& Stampfli, 2008). In contrast, the only data available on the age, petrology and geochemistry of the intrusive section of the Evros ophiolites refer to the Middle Jurassic Petrota gabbros and associated leucocratic dykes (Biggazzi et al. 1989; Frass et al. 1990) or have been recently communicated (Bonev \& Stampfli, 2005; Koglin et al. 2007; Magganas, 2007). The lack of data on the intrusive rocks makes it difficult to establish the extent of the tectono-magmatic section and thus the significance of these ophiolites. Therefore, understanding the petrology and geochemical signature of the various units of the Evros ophiolites is particularly important because they offer the key to reconstructing the setting in which the ophiolites formed.

This paper focuses on the plutonic rocks of the intrusive section and associated mafic dykes of the Evros ophiolites in Thrace, northeastern Greece. The aims of the study are: (1) to provide details of their 


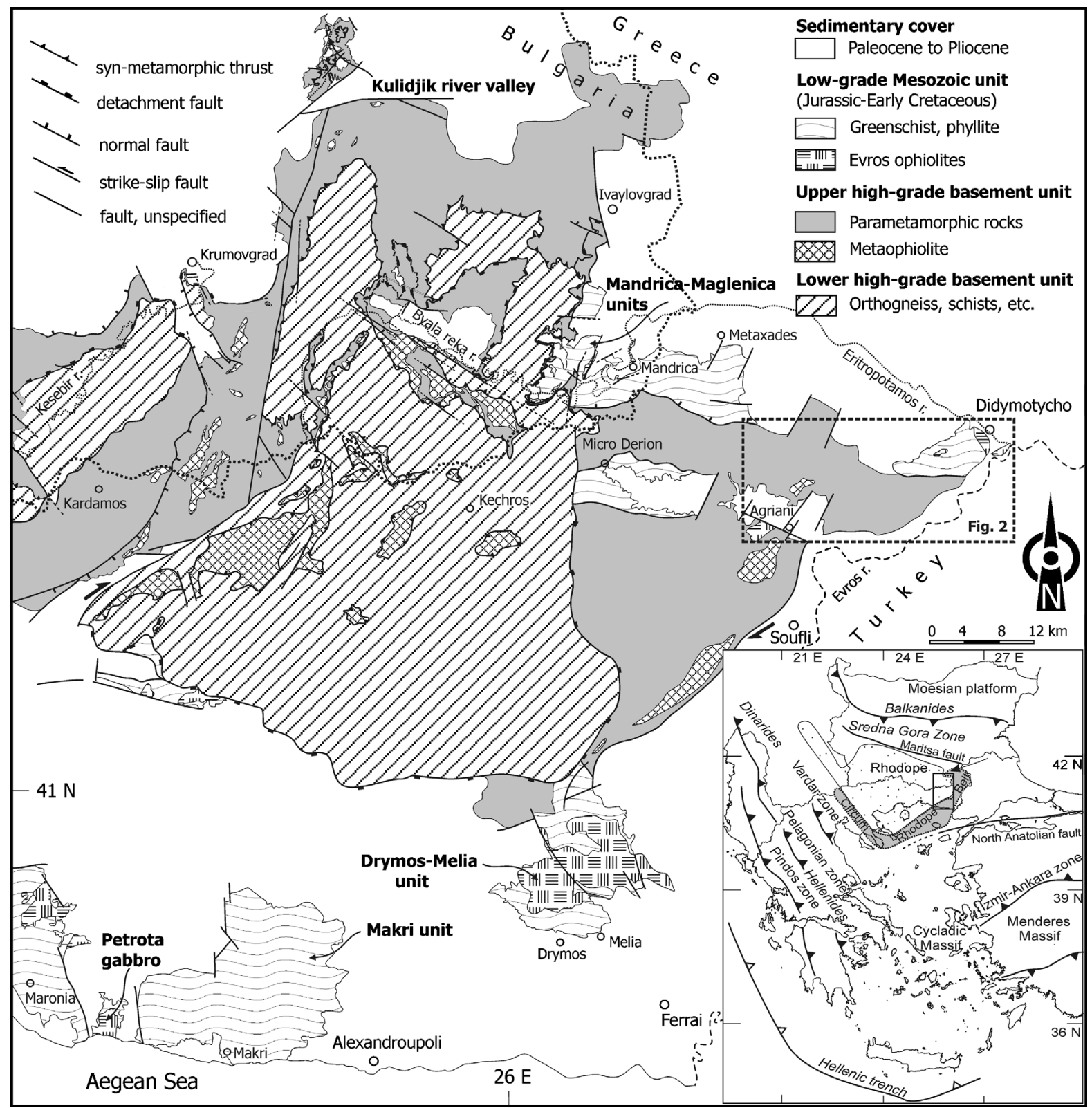

Figure 1. Tectonic map of the Eastern Rhodope-Thrace area (compiled after Papadopoulos \& Anastasiadis, 2002; Bonev, 2006), showing the main units and large-scale structures. Inset: tectonic framework of the Alpine system in the Aegean domain of the eastern Mediterranean region. Frame shows map area in Figure 2.

composition and tectonic setting, and (2) to compare the geochemistry of the plutonic rocks and dykes with data from the lavas of the extrusive sequence, in order to understand better the origin and evolution of the Evros ophiolites. We present field data, petrology and geochemical signatures of gabbroic and plagiogranite bodies and associated mafic dykes. Finally, we discuss their petrogenesis and tectonic significance for the tectono-magmatic section of the Evros ophiolites.

\section{Geological outline}

The Evros ophiolites are regarded as part of the Circum-Rhodope Belt (Kauffmann, Kockel \& Mollat, 1976; Kockel, Mollat \& Walther, 1977; Papanikolaou,
1997), found around the Rhodope massif, a major tectonic zone in the north Aegean region that extends through Bulgaria and Greece (Fig. 1, inset). To the north, the Rhodope massif is separated by a strike-slip fault from the Sredna Gora Zone, a Late Cretaceous volcanic arc. To the southwest, together with the SerboMacedonian massif, it is limited by the Vardar Suture Zone against the Hellenides (Fig. 1). The Rhodope massif is dominated by a metamorphic basement comprising pre-Alpine and Alpine (e.g. Lips, White \& Wijbrans, 2000; Liati, 2005) units of continental and oceanic affinities. The basement is intruded by Late Cretaceous to Early Miocene granitoids (Soldatos \& Christofides, 1986; Del Moro et al. 1988; Dinter et al. 1995; Peytcheva et al. 1999). Late 
Cretaceous-Paleocene to Miocene sediments (Ivanov \& Kopp, 1969; Boyanov \& Goranov, 2001) and Late Eocene-Oligocene volcanic successions (Innocenti et al. 1984; Harkovska, Yanev \& Marchev, 1989) represent cover sequences. The Rhodope massif is regarded as a S-directed nappe stack assembled in the hanging-wall of the N-dipping Cretaceous subduction zone located in the Vardar Zone (Ricou et al. 1998). The nappe stacking was accompanied and succeeded by crustal extension (Burg et al. 1996; Dinter, 1998; Krohe \& Mposkos, 2002; Bonev, Burg \& Ivanov, 2006; Bonev, 2006).

The tectonostratigraphic section of the eastern Rhodope-Thrace region comprises the following main units (Fig. 1): (1) a lower high-grade basement unit of continental affinity composed of orthogneisses with Variscan protolith ages (Peytcheva \& Quadt, 1995; Liati, 2005), (2) an upper lithologically heterogeneous high-grade basement unit of continental-oceanic affinity, enclosing arc tholeiitic and boninitic metaophiolites (Haydoutov et al. 2004) with Neoproterozoic protolith and Variscan metamorphic ages (Carrigan et al. 2003), (3) a low-grade unit of Mesozoic rocks (Jaranov, 1960; von Braun, 1968; Kopp, 1969) that includes the Evros ophiolites, and (4) a sedimentary unit of Tertiary cover sequences.

The high-grade basement units present a complex Alpine tectono-metamorphic history, temporarily bracketed between c. 149-61 Ma (Liati, 2005 and references therein), and cooling history between 45 $36 \mathrm{Ma}$ (Lips, White \& Wijbrans, 2000; Mukasa et al. 2003; Bonev, Marchev \& Singer, 2006) that relates to their extensional exhumation.

The low-grade unit outcrops are scattered, and lie tectonically on the high-grade basement as remnants of a former nappe (Boyanov et al. 1990) that has been reworked by Tertiary extensional tectonics (Bonev \& Stampfli, 2003). In the eastern Bulgarian Rhodope, it occurs in two main outcrop areas: along the Kulidjik river valley in the north, and in the Mandrica area in the south. In the Mandrica area, from the base to the top, the two lithostratigraphic units, the Mandrica and the Maglenica Group (Boyanov et al. 1990), were correlated with the Makri and Drymos-Melia units, respectively, in Thrace (Boyanov \& Russeva, 1989; Papadopoulos, Arvanitidis \& Zanas, 1989). Despite the discontinuous outcrop pattern of the lowgrade unit at a regional scale, the stratigraphy of all distinct units can be summarized as follows. The general stratigraphy comprises marbles, calc-schists, greenschists and phyllites at the base (e.g. Makri unit and Mandrica Group), overlain by an extrusive suite of the Evros ophiolites, which in turn are overlain by flysch (e.g. Drymos-Melia unit) and turbiditic sediments (lower Maglenica Group) at the top.

The intrusive suite of the Evros ophiolites, represented by a serpentinite in the Makri unit (Magganas, 2002) and the Petrota complex, occurs at a structurally deeper level (Fig. 1). The Petrota complex comprises tholeiitic to calc-alkaline affinity isotropic and cumulitic gabbros, gabbro-norites and anorthosites of assumed supra-subduction zone origin (Biggazzi et al. 1989). Apatite fission-track analysis of two samples from the Petrota gabbros gave ages of 140 and $161 \mathrm{Ma}$, respectively (Biggazzi et al. 1989), reflecting its cooling or emplacement history, whereas SHRIMP II zircon dating yielded a gabbro crystallization age of $169 \pm 2$ Ma (Koglin et al. 2007).

Palaeontological ages of the low-grade unit range from Upper Permian to Upper Cretaceous. The coherent strata yielded Middle Triassic to Lower Cretaceous fossils (Trikkalinos, 1955; Maratos \& Andronopoulos, 1964; Kopp, 1969; Boyanov \& Lipman, 1973; Tikhomirova, Boyanov \& Zagorchev, 1988; Boyanov et al. 1990; Dimadis et al. 1996; Dimadis \& Nikolov, 1997). Reworked Upper Permian and Middle-Upper Triassic clastics (Boyanov \& Budurov, 1979; Trifonova \& Boyanov, 1986; Bonev, 2005) and Upper Cretaceous fossils were reported in the upper levels of the lowgrade unit only in Bulgaria (Boyanov, Russeva \& Dimitrova, 1982), the latter fossils likely indicating a Campanian unconformity.

\section{Field data and structural features}

The study was conducted in the easternmost exposed part of the Evros ophiolites near the Greek-Turkish border (Fig. 2a). The local tectonostratigraphy is summarized in Figure $2 b$.

At Didymotycho, the $<180 \mathrm{~m}$ thick section of the Evros ophiolites mostly comprises massive to plagioclase and pyroxene-phyric mafic lava flows, and rare pillowed and hyaloclastic varieties of the extrusive sequence, overlain unconformably by Palaeogene clastics (reworked ophiolite) and reefal limestones. In the southwest, the mafic rocks are underlain by a highstrain zone of deformed shales intercalated with marble layers. This shear zone separates the ophiolitic unit from high-grade basement rocks underneath. A small, non-foliated plagiogranite stock (Lat. N 41 ${ }^{\circ} 19^{\prime} 34.8^{\prime \prime}$, Long. E $26^{\circ} 28^{\prime} 45.8^{\prime \prime}$ ) occurs below the mafic lavas, exposed in a recent quarry. Mutually intrusive relationships of the plagiogranite body with immediately overlying lavas are locally observed (Fig. 3a), while its base is not exposed. The plagiogranite intrusion in turn is cross-cut by numerous mafic dykes, with thickness up to $2 \mathrm{~m}$ (Fig. 3b). Most of the dykes are hydrothermally altered at the contacts. Less altered dykes do not show chilled margins, which suggests dyke emplacement while the plagiogranite intrusion was still hot. The dykes have a consistent orientation with a mean strike $\mathrm{N} 43.5^{\circ}$, dipping steeply $\left(80-65^{\circ}\right)$ mostly to the NW. The contacts of some dykes are traced by faults which preserve down-dip slickenside lineation, indicating dyke emplacement along NE-SWtrending normal faults, whose orientation equals that of the dykes (Fig. 2, stereoplot). In addition, small offsets of the dykes along striated oblique-slip faults marked 


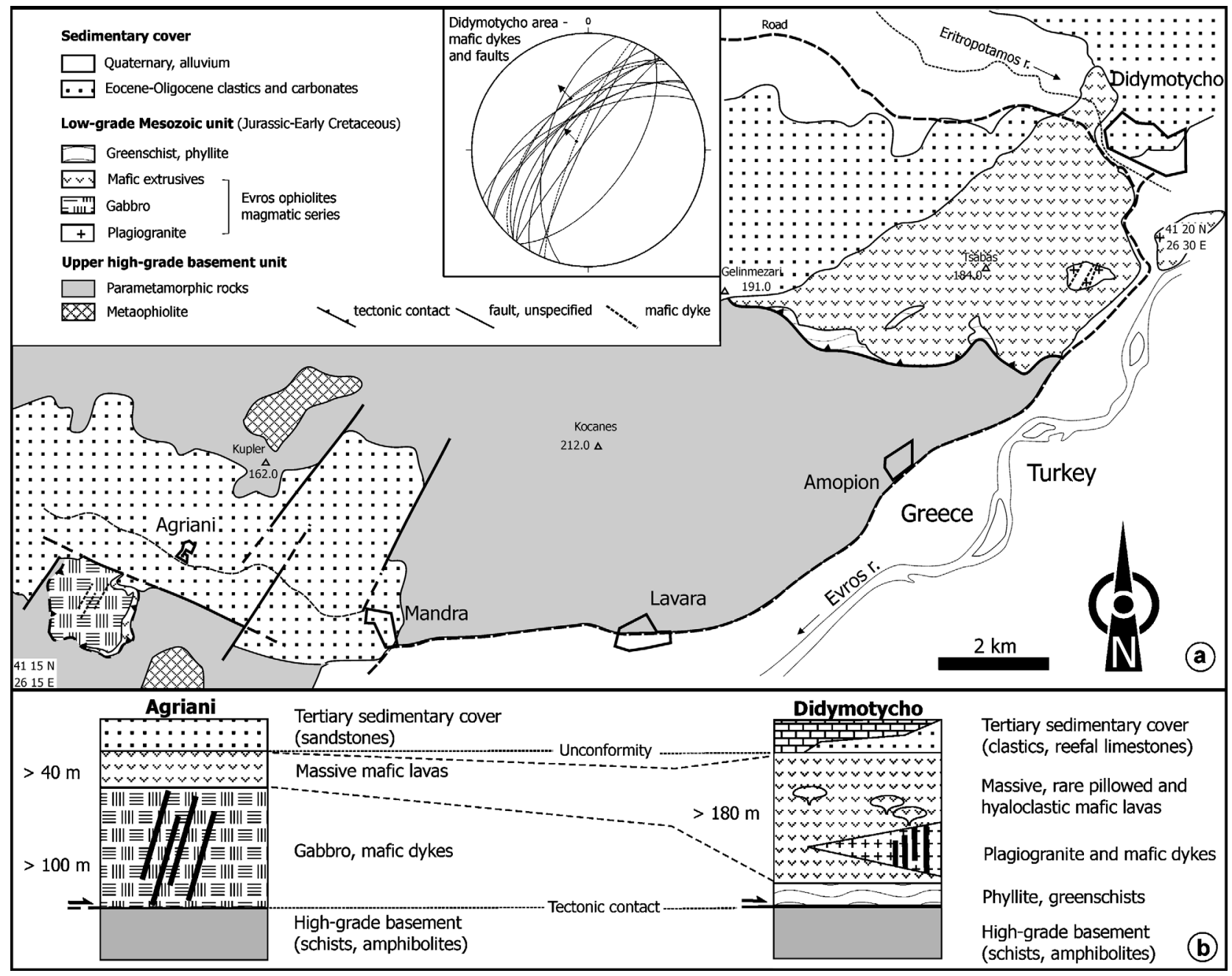

Figure 2. (a) Geological map of the area southwest of Didymotycho, Thrace, NE Greece. Stereoplot: lower hemisphere projection of mafic dykes (thick traces) and faults (dashed traces) with associated slickenside slip-lines (arrows). (b) Synthetic sections of the studied ophiolite localities.

by brecciate zones in the plagiogranite (Fig. 3c) imply a continuous stress regime after dyke emplacement.

Southwest of Agriani, a gabbroic body (Lat. $\mathrm{N} 41^{\circ} 16^{\prime} 36^{\prime \prime}$, Long. E $\left.26^{\circ} 16^{\prime} 17.8^{\prime \prime}\right)$ rests on the upper high-grade basement unit with poorly exposed basal tectonic contact. The gabbroic body lacks foliation, which makes it distinguishable from the meta-ophiolite body in the vicinity, and also from the metagabbro bodies that occur in the latter unit. The northern contact of the body is limited by a late fault, bounding in the southwest the small graben filled by Tertiary clastics, or in places the latter deposits unconformably overlie the gabbros. The gabbroic body is intruded by mafic dykes and overlain by a thin sheet flow of altered massive mafic lavas; this lava sheet can be considered as part of an up-section extrusive sequence of the ophiolites. The nearly vertical cross-cutting dykes trend NE-SW (mean strike $\mathrm{N} 35^{\circ}$ ), having an orientation consistent with the dykes that intrude the plagiogranite.

In both ophiolite occurrences, the steep orientation of the dykes, and evidence of their emplacement accompanied by fault displacements for those associated with the plagiogranite, suggest that the dykes have intruded in response to an NW-SE-oriented (present reference frame) crustal extensional regime.

\section{Petrography}

\section{4.a. Gabbro}

The gabbro is a mesocratic medium-grained rock comprising plagioclase, clinopyroxene, amphibole and very minor Fe-Ti oxides. The gabbro exhibits granular and poikilitic textures. Plagioclase forms either large euhedral laths or small crystals, the latter included in pyroxene and amphibole. Pyroxene occurs as subhedral to euhedral crystals intergrown with plagioclase, or is included within the primary brown-greenish amphibole. Secondary actinolite replaces pyroxene rims, or is present in interstices after primary amphibole. Both plagioclase and pyroxene are poikilitically enclosed in primary amphibole (Fig. 4a). The crystallization order is plagioclase $\rightarrow$ plagioclase + clinopyroxene $\rightarrow$ amphibole. Accessories are zircon, monazite and apatite. The gabbro has experienced a variable overprint of ocean floor hydrothermal 

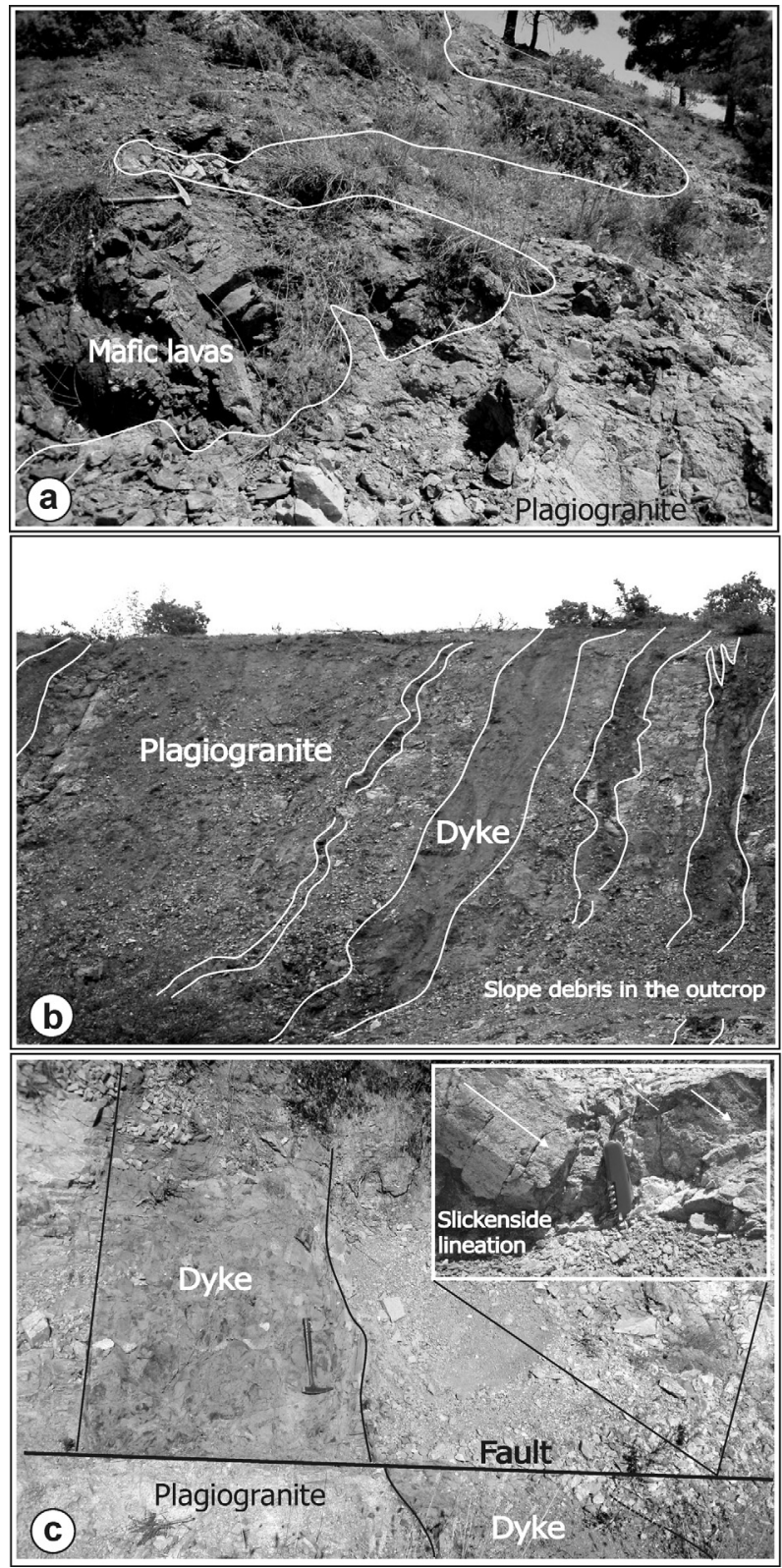

Figure 3. Field photographs of the plagiogranite and intruding mafic dykes. (a) Mutually intrusive contact between plagiogranite and mafic lavas. The hammer length is $34 \mathrm{~cm}$. (b) Mafic dykes intruding the plagiogranite. Width of field of view is $15 \mathrm{~m}$. (c) Mafic dyke offset along oblique-slip fault; inset: slickenside lineation on same fault surface.

alteration and/or weak greenschist metamorphism. Secondary phases are mostly chlorite after amphibole, and rarely epidote-group minerals.

\section{4.b. Plagiogranite}

The plagiogranite is a leucocratic rock composed of euhedral plagioclase modally predominant over subhedral to interstitial quartz and minor $(<10 \%)$ former ferro-magnesian mineral, with typical lack of alkali feldspar. Accessories include zircon and apatite. The medium-grained hypidiomorphic granular texture of the plagiogranite consists of mosaic intergrowths of the main mineral constituents. A 'myrmekitic' texture of vermicular quartz embayment into plagioclase or micrographic intergrowths of quartz and plagioclase common in this type of rocks is also observed (Fig. 4b), which suggests eutectic crystallization. Plagioclase is often altered to saussurite; chlorite, opaques and rarely epidote-group minerals have developed at the expense of pre-existing ferro-magnesian minerals, possibly amphibole. This indicates that hydrothermal alteration likely resulting from an ocean-floor water circulation has affected the plagiogranite rocks, or they have experienced weak greenschist metamorphism.

\section{4.c. Mafic dykes}

Mafic dykes include rock types that range from basalts, basaltic andesites to andesites as deduced from their textures, mineralogy and whole-rock compositions (see next Sections), and will be described separately according to their host rocks.

\section{4.c.1. Mafic dykes intruding the gabbro}

Basaltic andesite dykes display intersertal to intergranular and fine-grained porphyritic textures. The porphyritic ( $>10-15 \%$ phenocrysts) dykes consist of euhedral clinopyroxene and plagioclase microphenocrysts, set in a microlitic to intersertal groundmass composed of plagioclase, clinopyroxene, $\mathrm{Fe}-\mathrm{Ti}$ oxides, \pm glass (Fig. 4c). Phenocryst phases form occasionally glomeroporphyric clots. The intersertal to intergranular texture presents euhedral lath-shaped plagioclases and plagioclase microlites intergrown with euhedral to subhedral clinopyroxene microlites. The Fe-Ti minerals occupy spaces between plagioclase and clinopyroxene (Fig. 4d). The crystallization order is clinopyroxene $\rightarrow$ plagioclase + clinopyroxene $\rightarrow \mathrm{Fe}-$ Ti oxides. Alteration products include chlorite and rare thin calcite veinlets.

\section{4.c.2. Mafic dykes intruding the plagiogranite}

Basaltic, basaltic andesite and andesitic dykes display intersertal, porphyritic and amygdaloidal textures. Porphyritic textures present orthopyroxene and plagioclase microphenocrysts enclosed in a groundmass composed of microlites of the phenocryst phases, $\pm \mathrm{Fe}-\mathrm{Ti}$ oxides (Fig. 4e). Intersertal textures display euhedral plagioclase laths intergrown with clinopyroxene, both enclosed in microlitic matrix with disseminated $\mathrm{Fe}-\mathrm{Ti}$ oxides (Fig. 4f). Amygdaloidal textures are present in spilitized basaltic dykes, where the glassy groundmass is replaced by smectite and chlorite, and amygdales are filled with chlorite and quartz. Alteration products include chlorite pseudomorphs after pyroxene phenocrysts and within the groundmass. The crystallization order is pyroxene $\rightarrow$ plagioclase + pyroxene $\rightarrow \mathrm{Fe}-\mathrm{Ti}$ oxides. 

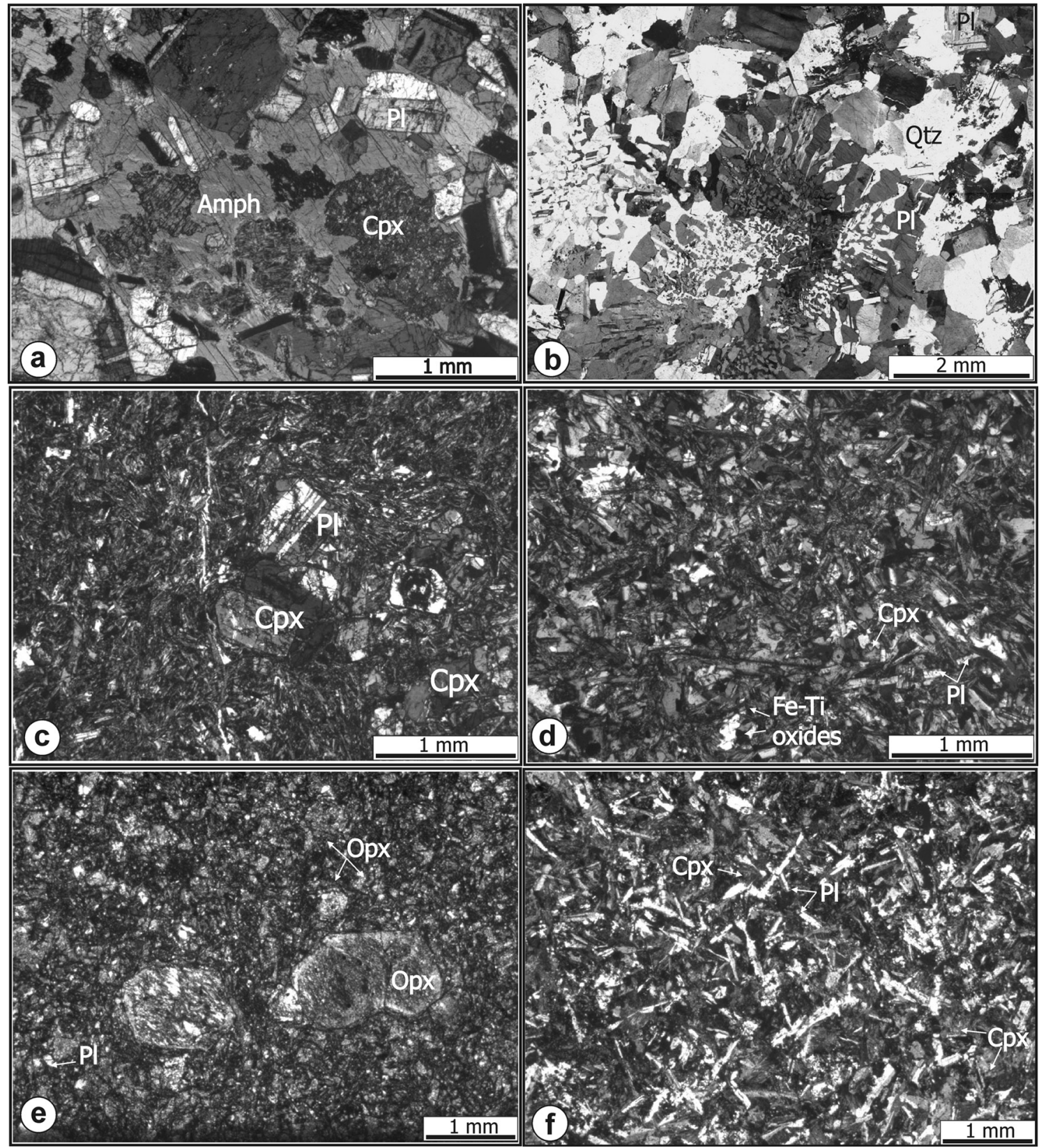

Figure 4. Microphotographs of the textural features. (a) Granular texture of gabbro with amphibole poikilitically enclosing plagioclase and pyroxene. (b) Granular texture (upper right in the photo) and 'myrmekitic' texture (centre) of plagiogranite, the latter presenting micrographic intergrowths of quartz and plagioclase and vermicular blebs of quartz within the plagioclase crystals. (c) Porphyritic texture of mafic dyke intruding the gabbro. (d) Intersertal-intergranular texture of mafic dyke intruding the gabbro. (e) Porphyritic texture of mafic dyke intruding the plagiogranite. (f) Intersertal texture of dyke intruding the plagiogranite. Abbreviations: Cpx clinopyroxene; Opx - clinopyroxene; Pl - plagioclase; Amph - amphibole; Qtz - quartz.

\section{Mineral chemistry}

\section{5.a. Analytical techniques}

Electron microprobe analyses were performed on a Cameca Camebax SX-50 equipped with four spectrometers and wavelength dispersive system in the University of Lausanne, Switzerland. Operating conditions were $20 \mathrm{nA}$ (pyroxenes, amphiboles) and $15 \mathrm{nA}$ (feldspars) focused beam at $15 \mathrm{kV}$ accelerating voltage, using natural standards. Representative chemical compositions and structural formulae of mineral phases are presented in tabular form in the Appendix and depicted therein in Figure A1
(Appendix available online as supplementary material at http://www.cambridge.org/journals/geo).

\section{5.b. Pyroxenes}

Orthopyroxenes in altered basaltic dykes that crosscut the plagiogranite vary from enstatite to ferrosilite $\left(\mathrm{Wo}_{0.60-0.17}, \mathrm{En}_{70.2-41.4}, \mathrm{Fs}_{57.9-29.5}\right)$. The orthopyroxenes have low $\mathrm{Cr}_{2} \mathrm{O}_{3} \quad(0.05-0.02 \mathrm{wt} \%), \quad \mathrm{MnO}(0.34$ $0.12 \mathrm{wt} \%)$ and very low $\mathrm{TiO}_{2}(0.04-0.07 \mathrm{wt} \%)$. The clinopyroxene is a ubiquitous phase in the gabbro and associated dykes. In terms of composition, the clinopyroxene is mainly $\mathrm{Mg}$-rich augite 
$\left(\mathrm{Wo}_{48.0-32.5}, \mathrm{En}_{67.5-46.1}, \mathrm{Fs}_{7.9-0.0}\right)$ in the gabbro, and augite $\left(\mathrm{Wo}_{43.7-38.04}, \mathrm{En}_{52.4-45.6}, \mathrm{Fs}_{16.3-4.0}\right)$ in the intruding dykes. The $\mathrm{Mg}$ no. ranges from $1.0-0.88$ in the gabbros and $0.93-0.74$ in the dykes. The high $\mathrm{Mg}$ no. of clinopyroxene is consistent with pyroxene compositions in other ophiolite complexes and island arcs (De Bari \& Coleman, 1989). All clinopyroxenes have low $\mathrm{Cr}_{2} \mathrm{O}_{3}$ content. The low Ti content of the clinopyroxenes likely reflects the degree of depletion of the mantle source (e.g. Pearce \& Norry, 1979). The low $\mathrm{Na}_{2} \mathrm{O}$ content of pyroxenes indicates a low pressure of crystallization and the subalkaline nature of the parental magma. Low $\mathrm{Ti} / \mathrm{Al}_{\text {tot }}$ ratios place clinopyroxenes in the arc-tholeiitic field of Leterrier et al. (1982), consistent with the formation in a subduction-related setting. Pyroxene thermometry (Lindsley, 1983) suggests higher temperatures of clinopyroxene crystallization in the gabbro and associated dykes, and a lower crystallization temperature for the dykes intruding the plagiogranite (Figure A1 of online Appendix).

\section{5.c. Amphiboles}

Primary amphiboles are magnesio-hornblende, tschermakitic hornblende and ferro-tschermakite (Figure A1 of online Appendix), whereas amphiboles replacing clinopyroxene and primary amphiboles are actinolite and actinolitic-tremolitic hornblende. Amphiboles have low $\mathrm{Cr}_{2} \mathrm{O}_{3}$ and $\mathrm{TiO}_{2}$ contents. The low $\mathrm{Al}$ contents of primary amphiboles imply low-pressure conditions (2.6-3.5 kbar: Anderson \& Smith, 1995) for amphibole crystallization. The majority of $\mathrm{Al}$ occurs as $\mathrm{Al}^{\mathrm{IV}}$, reflecting a high temperature of crystallization, also consistent with the crystallization temperature and composition of clinopyroxene in the gabbro.

\section{5.d. Plagioclases}

All plagioclases are unzoned. Plagioclase is bytownite $\left(\mathrm{An}_{89-78}\right)$ in the gabbro, but is more calcic $\left(\mathrm{An}_{99-74}\right)$ in associated dykes, reaching nearly pure anorthite. The high An contents are comparable to those of plagioclases in arc-related igneous rocks (Dupuy et al. 1982; Beard, 1986; De Bari \& Coleman, 1989), reflecting elevated magmatic dissolved water contents and high $\mathrm{Ca} / \mathrm{Ca}+\mathrm{Na}$ ratios (e.g. Arculus \& Wills, 1980; Sisson \& Grove, 1993; Panjasawatwong et al. 1997). Plagiogranite feldspars are pure albites $\left(\mathrm{An}_{0.5-0.2}\right)$. The plagioclases in the dykes that cross-cut the plagiogranite mainly have compositions of albiteoligoclase $\left(\mathrm{An}_{2-13}\right)$, with preserved more anorthite-rich compositions $\left(\mathrm{An}_{51}\right)$ in less evolved varieties.

\section{Whole-rock geochemistry}

\section{6.a. Analytical techniques and alteration}

Chemical analyses were determined at the University of Lausanne, Switzerland, and calibrated against both international and internal standards. Major and trace element concentrations were determined by Xray fluorescence (XRF) on fused discs and pressed pellets, respectively, using a Philips PW 2400 spectrometer. Rare-earth element (REE) concentrations were analysed by laser inductively coupled plasma mass spectrometry (LA-ICP-MS) on a Perkin-Elmer 6100 ELAN instrument on fused discs, using NBS612 standards. We present chemical data for a suite of gabbro samples (three) and associated dykes (three) and plagiogranite (four samples) and seven samples from the intruding dykes (Tables 1,2). A relatively high loss on ignition (LOI) in samples of the dykes associated with the plagiogranite attests to the variable degree of alteration of these rocks. We therefore use immobile trace elements (e.g. Nb, Ti, Zr, V, Hf, Ta, $Y$ ) and REE for the evaluation of petrological and geochemical signatures.

\section{6.b. Major and trace elements}

$\mathrm{SiO}_{2}$ abundances cover a narrow compositional range in the gabbro (46.4-48.8 $\mathrm{wt} \%$ ) and associated dykes $(53.2-55.1 \mathrm{wt} \%)$. Both are characterized by low $\mathrm{TiO}_{2}$ (0.18-0.49 wt \%) and total alkalis (av. $1.59 \mathrm{wt} \%$ in the gabbro, av. $2.49 \mathrm{wt} \%$ in the dykes). $\mathrm{MgO}$ contents range from $5.63-8.49 \mathrm{wt} \%$ in the dykes and 7.6$14.4 \mathrm{wt} \%$ in the gabbro (Fig. 5a). With the exception of gabbro sample AG2 (Table 1), Ni (17-88 ppm) and $\mathrm{Cr}(8-239 \mathrm{ppm})$ concentrations do not meet requirements for primary mantle melts $(\mathrm{Ni}>200 \mathrm{ppm}$, $\mathrm{Cr}>400 \mathrm{ppm}$ ), which together with the range of $\mathrm{Mg}$ no. (80-73) in the gabbro implies processes of fractional crystallization, more advanced in the dykes (Mg no. 70-55). With $\mathrm{Al}_{2} \mathrm{O}_{3}$ up to $20 \mathrm{wt} \%$ and $\mathrm{Sc}$ $<50 \mathrm{ppm}$, the gabbro differs from the magmatic rocks containing cumulitic plagioclase and pyroxene (e.g. Pearce, 1996). The gabbro and mafic dykes fall in the sub-alkaline basalt and andesite/basalt fields (Fig. 5b).

The dykes associated with the plagiogranite range from basalt to andesite $(41.4-64.5 \mathrm{wt} \%$, Table 2, Fig. 5b) and have higher $\mathrm{TiO}_{2}(0.23-1.03 \mathrm{wt} \%)$ than the dykes intruding the gabbro. In terms of trace elements, the plagiogranite-hosted dykes present higher concentrations of $\mathrm{Nb}$ (4-7 ppm), $\mathrm{Zr}$ (up to $57 \mathrm{ppm}$ ) and $\mathrm{Y}$ (up to $24 \mathrm{ppm}$ ), compared to the dykes intrusive into the gabbro (Table 2). Relative to $\mathrm{Ni}$ and $\mathrm{Cr}$, they have low abundances, except higher concentrations in the dyke sample Dd1 (Cr 467 ppm, Ni $141 \mathrm{ppm})$. The major and trace element characteristics of the dykes hosted by plagiogranites coupled with the range of $\mathrm{Mg}$ no. (62-36) indicates a more fractionated trend.

The mafic rocks are tholeiitic (Fig. 5c), but the dykes which intrude the gabbros show a boninitic affinity with $\mathrm{MgO}>8 \mathrm{wt} \%, \mathrm{SiO}_{2}>52 \mathrm{wt} \%$ and $\mathrm{TiO}_{2}<0.5 \mathrm{wt} \%$ (Le Maitre, 2002); this tholeiitic-transitional boninitic affinity is also evident in the $\mathrm{CaO} / \mathrm{Al}_{2} \mathrm{O}_{3} \mathrm{v} . \mathrm{Mg}$ no. diagram (Fig. 5a; Crawford, Falloon \& Green, 1989). 
Table 1. Whole-rock chemical analyses and CIPW normative composition of intrusive rocks in the Evros ophiolites, NE Greece

\begin{tabular}{|c|c|c|c|c|c|c|c|}
\hline $\begin{array}{l}\text { Sample } \\
\text { Rock type } \\
\text { Location }\end{array}$ & $\begin{array}{c}\text { AG1 } \\
\text { gb } \\
\text { Agr }\end{array}$ & $\begin{array}{c}\mathrm{AG} 2 \\
\mathrm{gb} \\
\mathrm{Agr}\end{array}$ & $\begin{array}{c}\text { AG3 } \\
\text { gb } \\
\text { Agr }\end{array}$ & $\begin{array}{c}\text { PG1 } \\
\text { pg } \\
\text { Dcho }\end{array}$ & $\begin{array}{c}\text { PG2 } \\
\text { pg } \\
\text { Dcho }\end{array}$ & $\begin{array}{c}\text { PG3 } \\
\text { pg } \\
\text { Dcho }\end{array}$ & $\begin{array}{c}\text { PG4 } \\
\text { pg } \\
\text { Dcho }\end{array}$ \\
\hline $\mathrm{SiO}_{2}$ & 46.36 & 47.69 & 48.84 & 78.07 & 77.65 & 78.21 & 76.37 \\
\hline $\mathrm{TiO}_{2}$ & 0.18 & 0.24 & 0.21 & 0.22 & 0.19 & 0.18 & 0.21 \\
\hline $\mathrm{Al}_{2} \mathrm{O}_{3}$ & 19.46 & 13.25 & 20.05 & 12.23 & 11.63 & 11.83 & 12.17 \\
\hline $\mathrm{Fe}_{2} \mathrm{O}_{3}$ & 5.30 & 7.09 & 5.36 & 0.86 & 1.82 & 1.47 & 2.75 \\
\hline $\mathrm{MnO}$ & 0.10 & 0.14 & 0.09 & 0.01 & 0.01 & 0.02 & 0.02 \\
\hline $\mathrm{MgO}$ & 10.21 & 14.35 & 7.61 & 0.44 & 0.35 & 0.38 & 0.45 \\
\hline $\mathrm{CaO}$ & 14.03 & 12.49 & 13.35 & 0.28 & 0.31 & 1.08 & 0.15 \\
\hline $\mathrm{Na}_{2} \mathrm{O}$ & 1.16 & 1.17 & 1.54 & 5.74 & 5.63 & 5.88 & 5.74 \\
\hline $\mathrm{K}_{2} \mathrm{O}$ & 0.14 & 0.17 & 0.60 & 0.25 & 0.16 & 0.07 & 0.13 \\
\hline $\mathrm{P}_{2} \mathrm{O}_{5}$ & 0.04 & 0.02 & 0.01 & 0.03 & 0.02 & 0.01 & 0.04 \\
\hline $\mathrm{Cr}_{2} \mathrm{O}_{3}$ & n.d. & 0.09 & 0.01 & n.d. & n.d. & n.d. & n.d. \\
\hline $\mathrm{NiO}$ & 0.01 & 0.04 & 0.01 & n.d. & n.d. & n.d. & n.d. \\
\hline LOI & 2.92 & 3.34 & 2.05 & 1.60 & 1.62 & 0.58 & 1.69 \\
\hline Total & 99.71 & 100.10 & 99.71 & 99.71 & 99.39 & 99.71 & 99.71 \\
\hline $\mathrm{Nb}$ & 1 & 2 & 1 & 4 & 6 & 6 & 5 \\
\hline $\mathrm{Zr}$ & 15 & 16 & 20 & 139 & 185 & 196 & 198 \\
\hline $\mathrm{Y}$ & 8 & 10 & 10 & 60 & 67 & 60 & 78 \\
\hline $\mathrm{Ta}$ & 0.78 & 0.38 & 0.87 & 5.26 & 4.32 & 8.05 & 3.27 \\
\hline $\mathrm{Rb}$ & 8 & 8 & 24 & 5 & 5 & 3 & 4 \\
\hline $\mathrm{Sr}$ & 214 & 110 & 212 & 35 & 41 & 86 & 24 \\
\hline $\mathrm{Ba}$ & 11 & 21 & 43 & 53 & 61 & 43 & 55 \\
\hline $\mathrm{U}$ & 2 & 2 & 2 & 2 & 2 & 2 & 2 \\
\hline Th & 2 & 2 & 2 & 3 & 4 & 4 & 5 \\
\hline $\mathrm{Pb}$ & 13 & 19 & 12 & 8 & 7 & 7 & 7 \\
\hline $\mathrm{Hf}$ & 4 & 2 & 8 & 4 & 3 & 1 & 3 \\
\hline $\mathrm{Sc}$ & 18 & 35 & 15 & 5 & 6 & 2 & 2 \\
\hline $\mathrm{Cr}$ & 40 & 467 & 66 & 8 & 11 & 9 & 9 \\
\hline $\mathrm{V}$ & 115 & 152 & 124 & 4 & 13 & 2 & 2 \\
\hline $\mathrm{Ni}$ & 74 & 234 & 88 & 4 & 3 & 4 & 4 \\
\hline $\mathrm{Ga}$ & 13 & 9 & 15 & 18 & 17 & 16 & 19 \\
\hline $\mathrm{Zn}$ & 37 & 58 & 28 & 23 & 18 & 15 & 33 \\
\hline $\mathrm{Cu}$ & 150 & 8 & 11 & 3 & 3 & 4 & 19 \\
\hline Co & 81 & 107 & 62 & 85 & 90 & 166 & 87 \\
\hline $\mathrm{La}$ & 0.67 & 0.86 & 1.15 & 9.80 & 7.77 & 8.05 & 11.39 \\
\hline $\mathrm{Ce}$ & 1.70 & 2.30 & 2.89 & 27.29 & 20.00 & 21.97 & 29.81 \\
\hline $\operatorname{Pr}$ & 0.27 & 0.33 & 0.38 & 4.08 & 3.15 & 3.38 & 4.72 \\
\hline $\mathrm{Nd}$ & 1.37 & 1.98 & 1.79 & 21.23 & 16.48 & 16.81 & 24.08 \\
\hline $\mathrm{Sm}$ & 0.35 & 0.89 & 0.77 & 6.30 & 5.20 & 5.53 & 6.75 \\
\hline $\mathrm{Eu}$ & 0.19 & 0.23 & 0.30 & 1.44 & 1.12 & 1.13 & 1.40 \\
\hline $\mathrm{Gd}$ & 0.56 & 1.01 & 0.77 & 8.51 & 8.94 & 7.52 & 10.35 \\
\hline $\mathrm{Tb}$ & 0.09 & 0.18 & 0.12 & 1.49 & 1.40 & 1.29 & 1.86 \\
\hline Dy & 0.77 & 1.17 & 1.15 & 8.84 & 10.77 & 9.70 & 13.86 \\
\hline Ho & 0.18 & 0.22 & 0.27 & 2.39 & 2.30 & 2.12 & 3.31 \\
\hline $\mathrm{Er}$ & 0.46 & 0.75 & 0.65 & 6.46 & 6.88 & 6.55 & 10.04 \\
\hline $\mathrm{Tm}$ & 0.03 & 0.11 & 0.12 & 0.90 & 1.16 & 0.93 & 1.50 \\
\hline $\mathrm{Yb}$ & 0.37 & 0.72 & 0.61 & 9.20 & 7.27 & 6.99 & 9.64 \\
\hline $\mathrm{Lu}$ & 0.06 & 0.13 & 0.07 & 0.90 & 1.07 & 1.35 & 1.44 \\
\hline Mg no. & 79.31 & 80.18 & 73.72 & 50.00 & 26.66 & 33.33 & 76.38 \\
\hline$(\mathrm{La} / \mathrm{Sm})_{\mathrm{n}}$ & 1.23 & 0.62 & 0.96 & 1.00 & 0.96 & 0.93 & 1.08 \\
\hline$(\mathrm{La} / \mathrm{Yb})_{\mathrm{n}}$ & 1.29 & 0.85 & 1.35 & 0.76 & 0.76 & 0.82 & 0.84 \\
\hline CIPW & Normative & Mineralogy & & & & & \\
\hline $\mathrm{Qz}$ & - & - & 2.04 & 42.55 & 43.22 & 40.95 & 41.67 \\
\hline Co & - & - & - & 2.08 & 1.68 & 0.15 & 2.41 \\
\hline Or & 0.83 & 1.00 & 3.54 & 1.48 & 0.94 & 0.41 & 0.77 \\
\hline $\mathrm{Ab}$ & 9.81 & 9.89 & 13.02 & 48.52 & 47.59 & 49.70 & 48.52 \\
\hline An & 47.45 & 30.39 & 46.00 & 1.19 & 1.41 & 5.29 & 0.49 \\
\hline Dy & 17.05 & 24.51 & 15.70 & - & - & - & - \\
\hline Hy & 13.00 & 20.82 & 11.64 & 1.09 & 0.87 & 0.94 & 1.12 \\
\hline $\mathrm{Ol}$ & 3.15 & 2.45 & - & - & - & - & - \\
\hline Il & 0.21 & 0.30 & 0.19 & 0.02 & 0.02 & 0.04 & 0.04 \\
\hline $\mathrm{Hm}$ & 5.31 & 7.10 & 5.37 & 0.86 & 1.82 & 1.47 & 2.76 \\
\hline $\mathrm{Ap}$ & 0.09 & 0.04 & 0.02 & 0.07 & 0.04 & 0.02 & 0.09 \\
\hline
\end{tabular}

Major elements (wt \%) determined by XRF; trace and rare earth elements (ppm) analysed by XRF and LA-ICP-MS. Abbreviations: gb - gabbro; pg - plagiogranite; n.d. - not detected. Agr - Agriani; Dcho - Didymotycho.

Plagiogranites have uniformly high $\mathrm{SiO}_{2}$ $(>76 \mathrm{wt} \%)$ and $\mathrm{Na}_{2} \mathrm{O}(>5 \mathrm{wt} \%)$ and very low $\mathrm{K}_{2} \mathrm{O} \quad(<0.16 \mathrm{wt} \%)$ contents (Table 1), typical of oceanic plagiogranites (Coleman \& Peterman, 1975; Coleman \& Donato, 1979; Aldiss, 1981; Pedersen \& Malpas, 1984), also having peraluminous 
Table 2. Whole-rock chemical analyses of mafic dykes in the Evros ophiolites, NE Greece

\begin{tabular}{|c|c|c|c|c|c|c|c|c|c|c|}
\hline $\begin{array}{l}\text { Sample } \\
\text { Rock type } \\
\text { Location }\end{array}$ & $\begin{array}{c}\text { AGd1 } \\
\text { band } \\
\text { Agr }\end{array}$ & $\begin{array}{c}\text { AGd2 } \\
\text { band } \\
\text { Agr }\end{array}$ & $\begin{array}{c}\text { AGd3 } \\
\text { band } \\
\text { Agr }\end{array}$ & $\begin{array}{l}\text { Dd1 } \\
\text { and } \\
\text { Dcho }\end{array}$ & $\begin{array}{c}\text { Dd2 } \\
\text { bas } \\
\text { Dcho }\end{array}$ & $\begin{array}{l}\text { Dd3 } \\
\text { band } \\
\text { Dcho }\end{array}$ & $\begin{array}{l}\text { Dd4 } \\
\text { band } \\
\text { Dcho }\end{array}$ & $\begin{array}{c}\text { Dd5 } \\
\text { and } \\
\text { Dcho }\end{array}$ & $\begin{array}{c}\text { Dd6 } \\
\text { bas } \\
\text { Dcho }\end{array}$ & $\begin{array}{l}\text { Dd7 } \\
\text { band } \\
\text { Dcho }\end{array}$ \\
\hline $\mathrm{SiO}_{2}$ & 53.21 & 53.52 & 55.06 & 60.59 & 41.41 & 50.74 & 49.71 & 64.51 & 42.35 & 48.22 \\
\hline $\mathrm{TiO}_{2}$ & 0.47 & 0.38 & 0.49 & 0.24 & 0.82 & 1.03 & 0.84 & 0.23 & 0.93 & 0.86 \\
\hline $\mathrm{Al}_{2} \mathrm{O}_{3}$ & 15.83 & 15.18 & 16.34 & 12.70 & 17.18 & 16.27 & 15.59 & 12.76 & 17.20 & 15.63 \\
\hline $\mathrm{Fe}_{2} \mathrm{O}_{3}$ & 7.84 & 7.09 & 9.15 & 9.15 & 24.06 & 13.04 & 14.85 & 7.52 & 20.58 & 19.12 \\
\hline $\mathrm{MnO}$ & 0.13 & 0.12 & 0.14 & 0.06 & 0.21 & 0.12 & 0.10 & 0.08 & 0.15 & 0.15 \\
\hline $\mathrm{MgO}$ & 7.53 & 8.49 & 5.63 & 7.60 & 7.92 & 5.25 & 5.85 & 5.13 & 5.84 & 7.23 \\
\hline $\mathrm{CaO}$ & 9.29 & 10.56 & 9.40 & 0.38 & 0.19 & 2.19 & 0.75 & 0.49 & 0.54 & 0.27 \\
\hline $\mathrm{Na}_{2} \mathrm{O}$ & 2.24 & 2.65 & 2.04 & 1.42 & 1.58 & 3.71 & 3.14 & 3.26 & 2.70 & 2.08 \\
\hline $\mathrm{K}_{2} \mathrm{O}$ & 0.34 & 0.10 & 0.11 & 0.11 & 0.03 & 0.11 & 0.14 & 0.30 & 0.15 & 0.13 \\
\hline $\mathrm{P}_{2} \mathrm{O}_{5}$ & 0.03 & 0.04 & 0.06 & 0.02 & 0.08 & 0.06 & 0.06 & 0.02 & 0.08 & 0.05 \\
\hline $\mathrm{Cr}_{2} \mathrm{O}_{3}$ & 0.03 & 0.05 & n.d. & 0.11 & n.d. & n.d. & n.d. & 0.08 & n.d. & n.d. \\
\hline $\mathrm{NiO}$ & 0.01 & 0.01 & n.d. & 0.02 & n.d. & n.d. & n.d. & 0.01 & n.d. & n.d. \\
\hline LOI & 3.08 & 1.68 & 2.23 & 7.53 & 6.94 & 7.37 & 9.16 & 6.17 & 9.13 & 6.41 \\
\hline Total & 100.02 & 99.87 & 100.65 & 99.93 & 100.42 & 99.89 & 100.19 & 100.56 & 99.54 & 100.15 \\
\hline $\mathrm{Nb}$ & 3 & 2 & 3 & 4 & 7 & 4 & 6 & 5 & 7 & 7 \\
\hline $\mathrm{Zr}$ & 39 & 40 & 42 & 17 & 46 & 57 & 48 & 16 & 56 & 43 \\
\hline $\mathrm{Y}$ & 14 & 14 & 18 & 11 & 18 & 24 & 20 & 12 & 23 & 14 \\
\hline $\mathrm{Ta}$ & 0.83 & 1.00 & 0.32 & 0.44 & 0.15 & 0.30 & 0.15 & 0.33 & 0.25 & 1.02 \\
\hline $\mathrm{Rb}$ & 15 & 3 & 4 & 6 & 2 & 4 & 5 & 16 & 6 & 6 \\
\hline $\mathrm{Sr}$ & 190 & 120 & 132 & 26 & 13 & 91 & 61 & 73 & 34 & 17 \\
\hline $\mathrm{Ba}$ & 44 & 41 & 23 & 42 & 9 & 68 & 61 & 77 & 34 & 20 \\
\hline $\mathrm{U}$ & 3 & 2 & 2 & 2 & 2 & 2 & 3 & 2 & 2 & 2 \\
\hline Th & 3 & 3 & 3 & 2 & 2 & 3 & 3 & 2 & 2 & 3 \\
\hline $\mathrm{Pb}$ & 14 & 15 & 16 & 32 & 26 & 12 & 50 & 56 & 33 & 30 \\
\hline $\mathrm{Hf}$ & 3 & 5 & 4 & 6 & 2 & 7 & 4 & 3 & 6 & 3 \\
\hline $\mathrm{Sc}$ & 33 & 25 & 35 & 107 & 169 & 102 & 129 & 78 & 166 & 132 \\
\hline $\mathrm{Cr}$ & 145 & 239 & 20 & 467 & 31 & 35 & 28 & 328 & 33 & 29 \\
\hline V & 221 & 192 & 229 & 301 & 361 & 402 & 344 & 239 & 400 & 367 \\
\hline $\mathrm{Ni}$ & 59 & 79 & 17 & 141 & 15 & 10 & 16 & 74 & 13 & 14 \\
\hline $\mathrm{Ga}$ & 15 & 13 & 16 & 15 & 34 & 19 & 18 & 12 & 27 & 23 \\
\hline $\mathrm{Zn}$ & 55 & 50 & 56 & 113 & 166 & 55 & 177 & 316 & 159 & 118 \\
\hline $\mathrm{Cu}$ & 66 & 88 & 37 & 12 & 2 & 6 & 4 & 102 & 3 & 2 \\
\hline $\mathrm{Co}$ & 65 & 78 & 80 & 71 & 105 & 74 & 59 & 60 & 92 & 96 \\
\hline $\mathrm{La}$ & 2.32 & 2.91 & 2.43 & 1.16 & 9.58 & 2.97 & 3.11 & 1.24 & 5.74 & 1.65 \\
\hline $\mathrm{Ce}$ & 6.40 & 6.29 & 5.86 & 1.20 & 24.77 & 7.17 & 4.84 & 1.41 & 11.04 & 4.49 \\
\hline $\operatorname{Pr}$ & 0.96 & 0.78 & 0.88 & 0.41 & 3.66 & 1.14 & 1.40 & 0.45 & 2.36 & 0.85 \\
\hline $\mathrm{Nd}$ & 3.78 & 3.28 & 4.13 & 2.94 & 20.37 & 6.29 & 7.87 & 2.70 & 12.61 & 4.57 \\
\hline $\mathrm{Sm}$ & 1.09 & 1.02 & 1.29 & 0.96 & 5.94 & 2.34 & 3.23 & 1.01 & 4.11 & 2.08 \\
\hline $\mathrm{Eu}$ & 0.49 & 0.36 & 0.57 & 0.39 & 1.04 & 0.69 & 0.76 & 0.18 & 0.87 & 0.25 \\
\hline Gd & 1.74 & 1.27 & 1.97 & 1.12 & 7.09 & 3.08 & 2.94 & 1.03 & 4.77 & 1.97 \\
\hline $\mathrm{Tb}$ & 0.22 & 0.29 & 0.34 & 0.20 & 1.00 & 0.56 & 0.50 & 0.19 & 0.86 & 0.36 \\
\hline Dy & 1.78 & 2.01 & 2.27 & 1.79 & 6.52 & 3.89 & 3.33 & 1.30 & 5.80 & 2.58 \\
\hline Ho & 0.33 & 0.36 & 0.51 & 0.25 & 1.11 & 0.84 & 0.89 & 0.28 & 1.07 & 0.61 \\
\hline $\mathrm{Er}$ & 1.03 & 1.30 & 1.60 & 1.24 & 3.19 & 2.46 & 2.12 & 0.78 & 3.66 & 1.58 \\
\hline $\mathrm{Tm}$ & 0.05 & 0.20 & 0.26 & 0.16 & 0.38 & 0.25 & 0.36 & 0.08 & 0.53 & 0.30 \\
\hline $\mathrm{Yb}$ & 1.05 & 0.79 & 1.45 & 0.84 & 2.60 & 2.21 & 1.94 & 1.07 & 3.16 & 2.14 \\
\hline $\mathrm{Lu}$ & 0.16 & 0.18 & 0.17 & 0.15 & 0.42 & 0.31 & 0.34 & 0.16 & 0.55 & 0.26 \\
\hline Mg no. & 65.49 & 70.46 & 54.94 & 62.25 & 39.43 & 44.36 & 43.80 & 57.46 & 35.91 & 42.82 \\
\hline $\mathrm{La} / \mathrm{Sm}_{\mathrm{n}}$ & 1.37 & 1.84 & 1.21 & 0.77 & 1.04 & 0.81 & 0.62 & 0.79 & 0.90 & 0.51 \\
\hline $\mathrm{La} / \mathrm{Yb}_{\mathrm{n}}$ & 1.58 & 2.64 & 1.20 & 0.98 & 2.64 & 0.96 & 0.91 & 0.83 & 1.30 & 0.55 \\
\hline
\end{tabular}

Major elements (wt \%) determined by XRF; trace and rare earth elements (ppm) analysed by XRF and LA-ICP-MS.

Abbreviations: bas - basalt; band - basaltic andesite; and - andesite; n.d. - not detected. Agr - Agriani; Dcho - Didymotycho.

character $\left(\mathrm{Al}_{2} \mathrm{O}_{3}<12.5 \mathrm{wt} \%, \mathrm{ACNK}=1.017-1.258\right)$ following a trondhjemite trend (Fig. 5c). On account of the modal content, mineralogy and major element chemistry, the plagiogranites are classified as high silica-low alumina trondhjemites. In terms of trace elements, the plagiogranites exhibit low $\mathrm{Rb}$ and $\mathrm{Sr}$ concentrations, and $\mathrm{Ba}$ and $\mathrm{Nb}$ contents relatively low for granitoids (Table 1). Other incompatible elements such as Zr (138-196 ppm) and Y (60-78 ppm) have contents in the range known for the oceanic crust acid differentiates.

In summary, the major and trace element characteristics of the mafic rocks classify them as low-K and low- to very low-Ti tholeiitic-transitional boninitic gabbro and basalts to andesites with transitional tholeiitic-boninitic and boninitic affinity, whereas plagiogranites present the oceanic character of high silica trondhjemites.

\section{6.c. Rare-earth elements patterns}

Chondrite-normalized REE patterns of plagiogranite samples show flat light rare earth element (LREE) $\left((\mathrm{La} / \mathrm{Sm})_{\mathrm{n}}=0.99\right)$ and variably fractionated heavy rare earth element $(\mathrm{HREE})\left((\mathrm{La} / \mathrm{Yb})_{\mathrm{n}}=0.79\right)$ profiles, with pronounced negative $\mathrm{Eu}$ anomaly indicating plagioclase involvement during fractionation and/or partial melting (Fig. 6a). The gabbro samples display nearly 

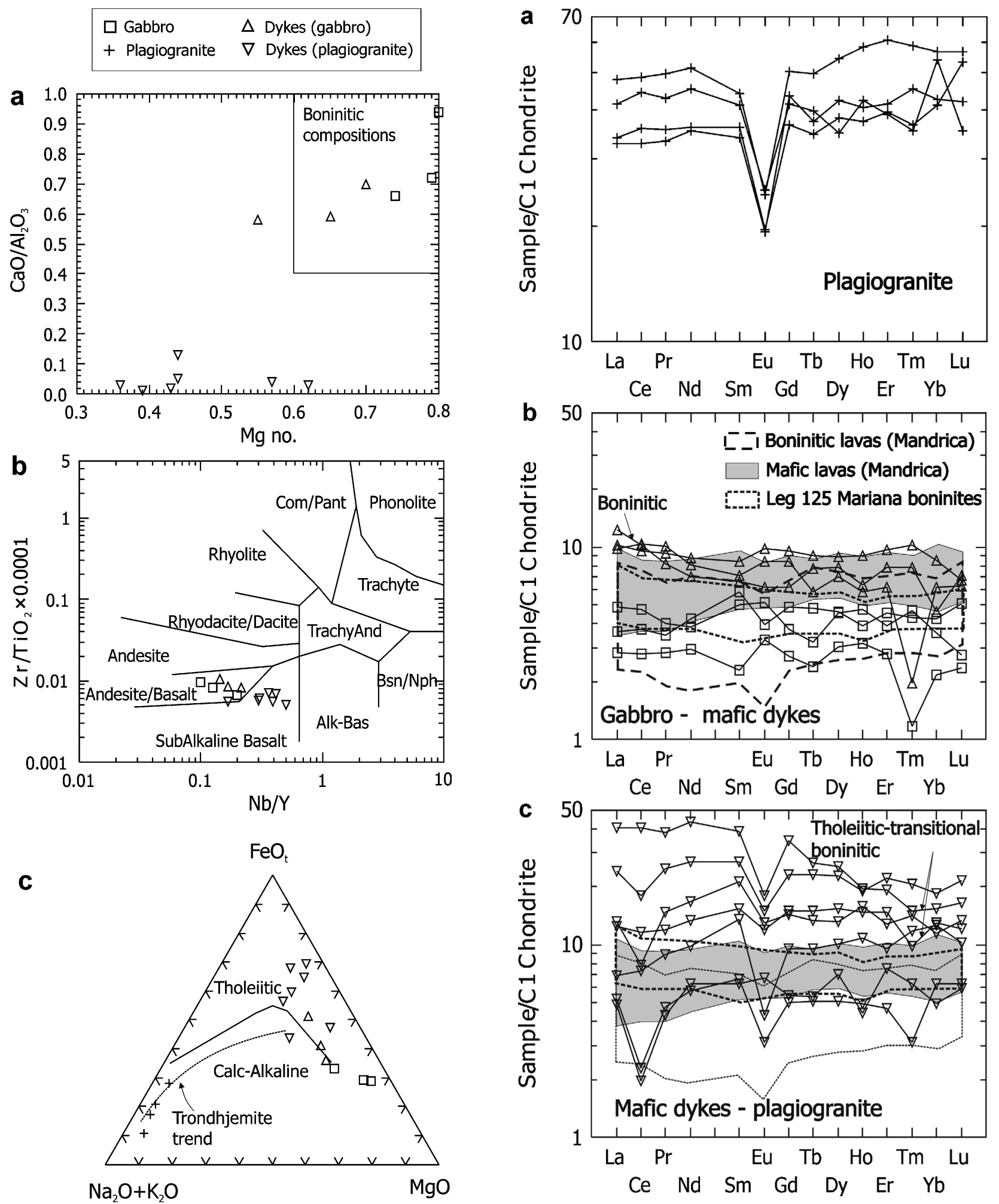

Figure 5. Classification and geochemical affinity of the plutonic rocks and associated mafic dykes. (a) $\mathrm{CaO} / \mathrm{Al}_{2} \mathrm{O}_{3}$ v. $\mathrm{Mg}$ no. diagram (after Crawford, Falloon \& Green, 1989) for the mafic rocks; (b) mafic rocks plotted on $\mathrm{Zr} / \mathrm{TiO}_{2} \mathrm{v}$. Nb/Y diagram (after Winchester \& Floyd, 1977); (c) samples plotted on AFM diagram (after Irvine \& Baragar, 1971); trondhjemite trend after Barker $\&$ Arth (1976).

flat LREE profiles $\left((\mathrm{La} / \mathrm{Sm})_{\mathrm{n}}=0.93\right)$, with positive and negative $\mathrm{Eu}$ anomalies indicating accumulation or removal of plagioclase, respectively, and fractionated HREE patterns $\left((\mathrm{La} / \mathrm{Yb})_{\mathrm{n}}=1.16\right)$ (Fig. 6b). The dykes associated with the gabbro present higher

Figure 6. Chondrite-normalized REE patterns for the plutonic rocks and mafic dykes. For symbols in this and the following figures, see Figure 5. Normalization values after Sun \& McDonough (1989). The fields of mafic tholeiitic basalticandesitic lavas (shaded), boninitic lavas (long dashed) from the Mandrica area (after Bonev \& Stampfli, 2008) and boninites from Leg 125 (short dashed) (after Pearce et al. 1992) are given for comparison in (b) and (c).

REE abundances compared to the gabbro, having slightly LREE enriched and fractionated HREE patterns $\left((\mathrm{La} / \mathrm{Sm})_{\mathrm{n}}=1.47,(\mathrm{La} / \mathrm{Yb})_{\mathrm{n}}=1.81\right)$. These dykes show slightly U-shaped REE profiles that are 
characteristic of boninites (Fig. 6b). Mafic dykes intrusive into the plagiogranite present systematic variations in the magnitude of REE enrichment and fractionated sub-parallel profiles (Fig. 6c). Most of these dykes have negative Ce anomaly and almost all possess a pronounced negative $\mathrm{Eu}$ anomaly, indicating removal of plagioclase by fractional crystallization or partial melting of a rock in which feldspar is retained in the source. In terms of REE abundances, the negative Eu anomaly and HREE fractionation, the dykes display patterns comparable to that shown by the host plagiogranite.

A multi-element spider diagram of plagiogranite samples normalized to normal mid-ocean ridge basalt (N-MORB) displays large-ion lithophile element (LILE) enrichment relative to high-field strength elements (HFSE). Pronounced P, Sr and Ti negative anomalies and lower magnitude negative $\mathrm{Nb}$ and $\mathrm{Zr}$ anomalies, but at higher abundances relative to $\mathrm{N}$ MORB, characterize the plagiogranites (Fig. 7a). The patterns of the plagiogranite samples normalized to oceanic ridge granite (ORG; Pearce, Harris \& Tindle, 1984) are similar in terms of $\mathrm{Nb}$ and $\mathrm{Zr}$ anomalies compared to N-MORB profiles, but significantly depart from typical ORG. LILE-enrichment, variable HFSE and REE depletion relative to N-MORB is well defined in the profiles of the gabbro and associated dykes, defining high LILE/HFSE ratios characteristic for island arc-related lavas (Fig. 7b). Both mafic rock types show negative $\mathrm{Ti}, \mathrm{Nb}, \mathrm{Ce}$ and $\mathrm{P}$ anomalies and positive $\mathrm{Th}, \mathrm{Pb}, \mathrm{Hf}$ and $\mathrm{Y}$ anomalies. N-MORB-normalized profiles of the dykes hosted by plagiogranite display similar patterns compared to samples of the gabbro and associated dykes, having less pronounced HFSE and HREE depletion relative to N-MORB and a negative Ta anomaly (Fig. 7c).

\section{Tectonic setting discrimination}

The trace element discrimination diagrams define an island arc setting for the gabbro and associated dykes, as well as for the dykes cross-cutting the plagiogranite (Fig. 8a, b). All mafic rocks plot in the volcanic arc field in the Th/Hf/Ta diagram of Wood (1980) and occupy island arc and boninite $(\mathrm{Ti} / \mathrm{V}$ ratio $<10)$ fields on the Ti/V diagram of Shervais (1982). Consequently, the ophiolitic rocks clearly display a strong signature of island arc tholeiitic (IAT) and boninitic affinity magmatic products. Plagiogranite samples fall in the ORG field (Fig. 8c), overlapping the field of suprasubduction type Troodos plagiogranites, and plot close to Guevgueli trondhjemites and plagiogranite samples reported by Magganas (2007).

\section{Discussion}

\section{8.a. Petrogenesis}

The petrography and mineral chemistry of the gabbro reveal textures and compositions which, combined with its geochemistry, are compatible with an isotropic
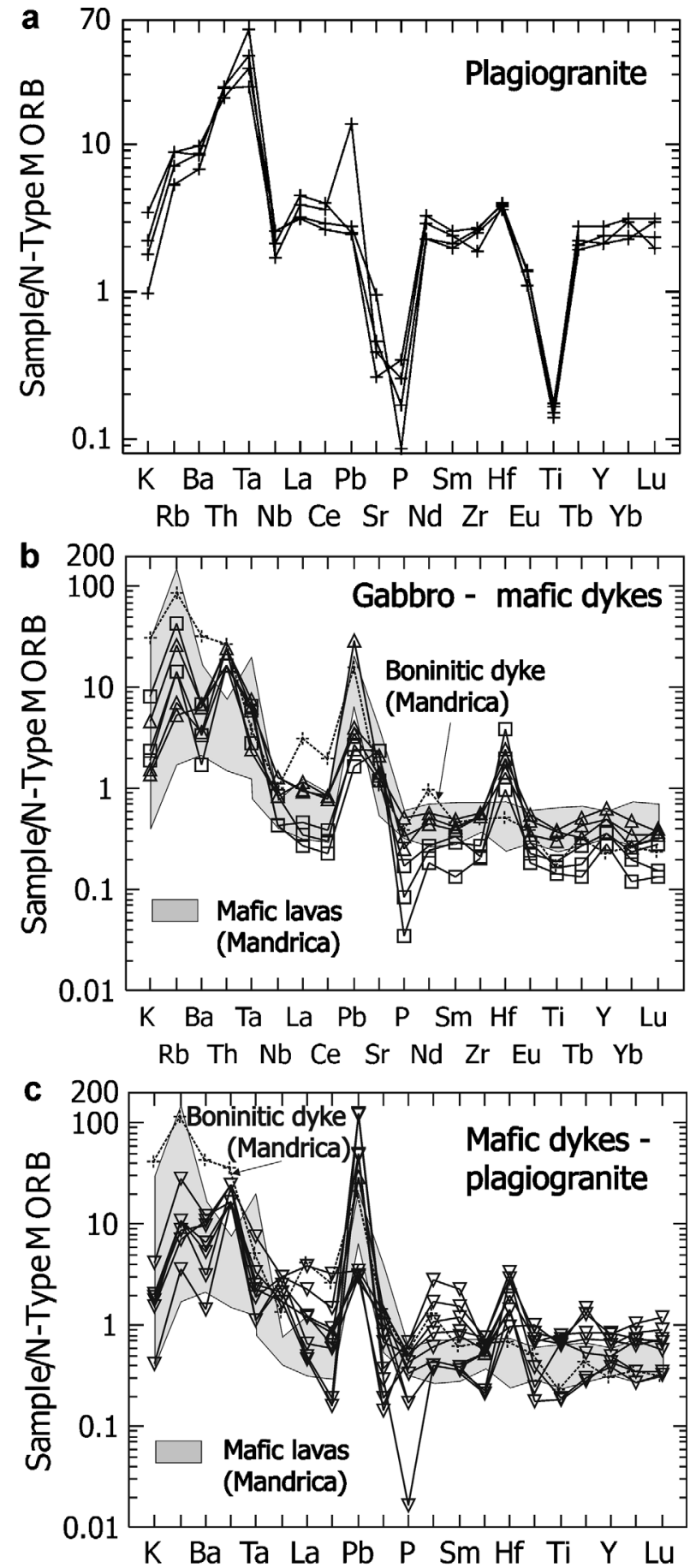

$\mathrm{Rb}$ Th $\mathrm{Nb} \mathrm{Ce} \mathrm{Sr} \mathrm{Nd} \mathrm{Zr} \mathrm{Eu} \mathrm{Tb} \mathrm{Yb}$

Figure 7. N-MORB normalized diagrams for the plutonic rocks and mafic dykes. Normalization values after Sun \& McDonough (1989). The envelope of mafic lavas (shaded) and a boninitic dyke (dashed line) from the Mandrica area (after Bonev \& Stampfli, 2008) are shown for comparison in (b) and (c).

gabbro. The high Mg no. and major element contents of the gabbro demonstrate a slightly differentiated nature, whose trace elements and REE patterns indicate N-MORB-depleted REE and HFSE characteristics (Table 1; Figs 6, 7). Thus, the gabbro chemistry, the high LILE/HFSE ratio and IAT signature (Figs 7,8) are consistent with derivation from a refractory mantle source in a subduction zone setting. Its origin by fluid-assisted low-pressure partial melting of depleted mantle peridotite above subducting slab is indicated 
a

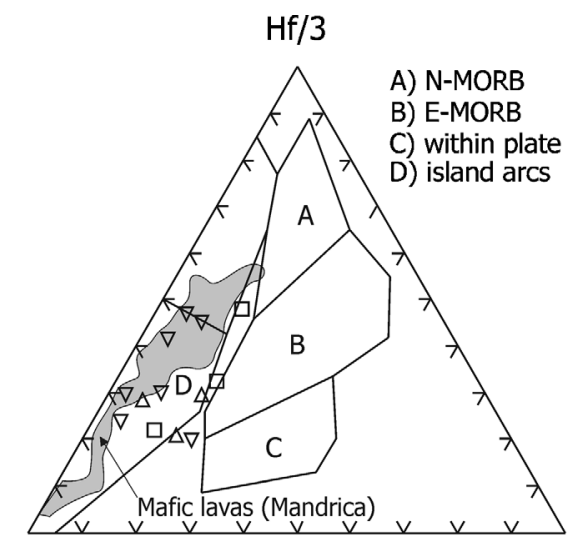

Th

$\mathrm{Ta}$
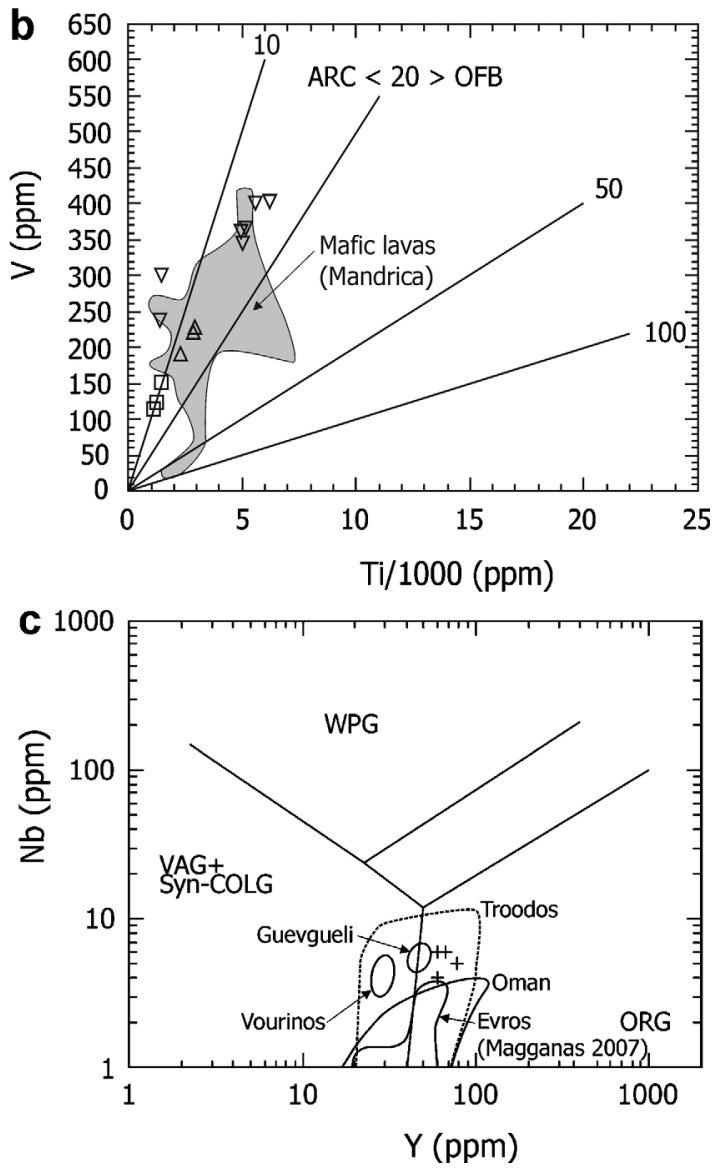

Figure 8. Trace element discrimination diagrams for the plutonic rocks and mafic dykes. (a) Th/Ta/Hf diagram (after Wood, 1980). (b) Ti v. V diagram (after Shervais, 1982). The field of mafic lavas from the Mandrica area (after Bonev \& Stampfli, 2008) is given for comparison in (a) and (b). (c) $\mathrm{Nb}$ v. Y diagram with fields of the plagiogranites in Vourinos, Troodos and Oman ophiolites (after Pearce, Harris \& Tindle, 1984); those for Guevgueli trondhjemites and Evros plagiogranites, respectively, are from Hatzipanagiotu \& Tsikouras (1999) and Magganas (2007).

by the presence of amphibole, together with the inferred thermo-barometric range for amphibole and clinopyroxene crystallization (Fig. 9a; Fig. A1 of online Appendix).

The dykes intruded into the gabbro exhibit phenocryst mineralogy comparable to the gabbro, having more evolved compositions and differentiated nature (Table 2; Fig. A1 of online Appendix), less depleted
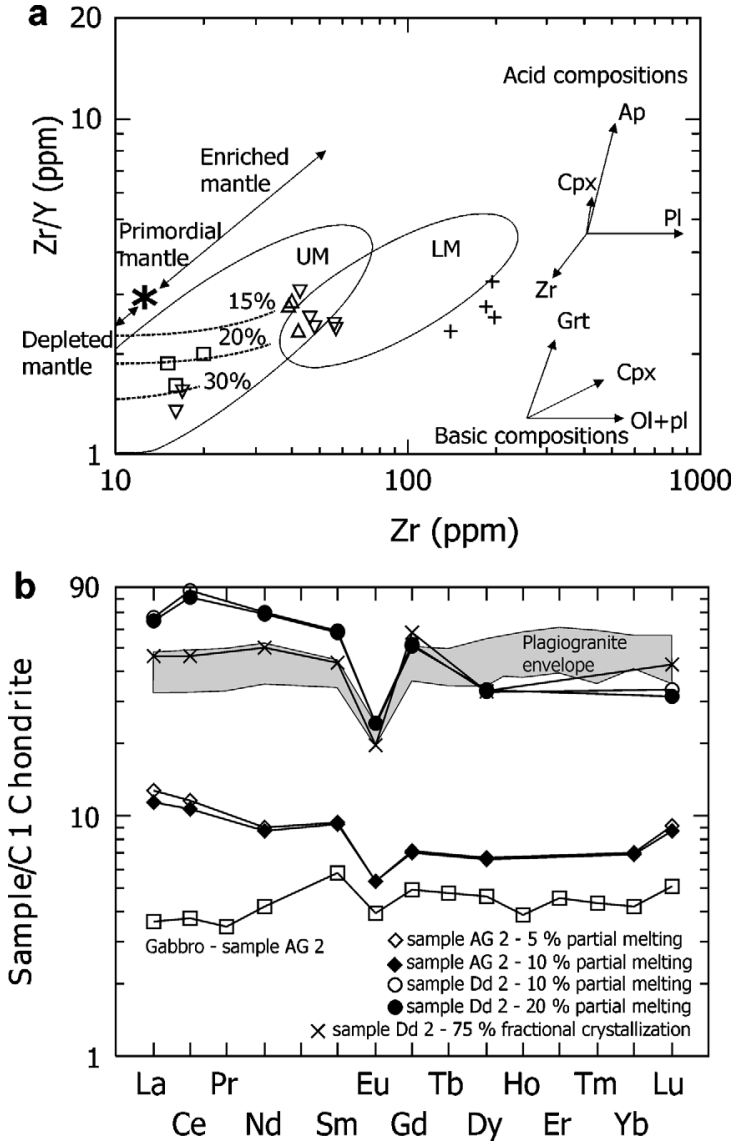

Figure 9. (a) $\mathrm{Zr} / \mathrm{Y}$ v. $\mathrm{Zr}$ diagram with partial melting and fractionation trends after Pearce \& Norry (1979) for the gabbro and mafic dykes. UM - upper metavolcanics and LM lower metavolcanics fields after Magganas (2002). Fractionation vectors for acid compositions after Floyd, Yaliniz \& Göncüoğlu (1998). (b) Model REE patterns of plagiogranite generation by fractionation and partial melting of mafic dyke and gabbro 'parents', compared with the range of chondrite-normalized plagiogranite compositions. Distribution coefficients are after compilation by Rollinson (1993).

to N-MORB REE and HFSE patterns compared to the gabbro and analogous arc-related signature. However, these boninitic dykes post-date the gabbro and account for a distinct slightly LREE-enriched mantle source (Figs 5, 6). The LILE and LREE enrichment as recorded in boninitic dykes has been shown to be a chemical fingerprint of a slab-derived component in subduction systems (e.g. Cameron, Nisbet \& Dietrich, 1979; Pearce, Lippard \& Roberts, 1984; McCulloch \& Gamble, 1991). Although slight chemical differences between the gabbro and boninitic dykes in terms of REE may account for the different parental magma or various degrees of melting, the shape of their trace element patterns is compatible with derivation from a common mantle source region, which likely experienced successive melt extraction. The very low Ti content in pyroxenes in the gabbro and boninitic dykes suggests that earlier melting events removed Ti from the pyroxenes (e.g. Hébert \& Laurent, 1990) and from the mantle source, respectively, which is consistent with their low-Ti character and pyroxene composition similar to boninites (Beccaluva et al. 1989). The 
consistence in composition of the source region for the gabbro and boninitic dykes is substantiated by the linear trend of major oxides, V/Ti ratio and HFSE-HREE depletion (Figs 5, 7, 8). The gabbro displays transitional boninitic affinity comparable to the boninitic dykes (Figs 5, 6) and low-silica high-Ca boninites of the Bonin-Mariana fore-arc (Fig. 6; Pearce et al. 1992).

The dykes associated with the plagiogranite share the general chemical features of the gabbro and boninitic dykes (Fig. 7). The most striking differences, however, are a low-Ca and Fe-rich character, more evolved compositions, extended $\mathrm{Mg}$ no. and variable $\mathrm{Zr}$ contents, attesting to their distinctly differentiated nature (Table 2; Fig. 9a). The differentiation processes in the dykes are indicated by a negative Eu anomaly, which together with $\mathrm{Sr}$ depletion implies fractionation involving removal of Ca-rich plagioclase, as well as apatite and Fe-Ti oxides. Systematic REE enrichment and the lesser extent of HFSE depletion in these dykes indicate less depletion in incompatible elements and an REE-enriched mantle source compared to the gabbro and boninitic dykes. This source, in view of LILE and REE enrichment, appears substantially modified by slab-derived fluids, thus the plagiogranite-hosted dykes document a more pronounced subduction-related component. In terms of the higher concentrations of $\mathrm{Nb}, \mathrm{Zr}$ and $\mathrm{Y}$ (Table 2; Fig. 9a), ascribed to a mantle component, these dykes are products of more fertile melts of a less refractory mantle.

All studied mafic rocks are notable by their negative Ce anomalies (Figs 6, 7), which are regarded as resulting from the subduction of sediments in arc systems (Hole et al. 1984; Elliott et al. 1997; Woodhead, Eggins \& Johnson, 1998). In the plagiogranite-hosted dykes, the Ce depletion apparently relates to a sediment contribution to the slab-derived component in their mantle source. The mafic rocks have high Hf contents and $\mathrm{Hf}_{\mathrm{n}} / \mathrm{Nd}_{\mathrm{n}}$ ratio, both pertinent to proto-arc magma genesis (Murton et al. 1992; Pearce et al. 1999).

Overall, the IAT-boninitic signature, the high LILE/HFSE ratios, the negative $\mathrm{Ta}-\mathrm{Nb}-\mathrm{Ti}$ anomalies and detectable slab-derived components of the studied mafic rocks, are all features typical for arc-related petrogenesis. Their trace element and REE chemistry indicates derivation from a more depleted mantle source than that of typical N-MORB, accounting for the successive melt extraction in the source region, a significant involvement of sediments in the subduction zone component during magma genesis and differentiation processes involving pyroxene, plagioclase, apatite, zircon and $\mathrm{Fe}-\mathrm{Ti}$-oxide fractionation from the mantle source en route to the surface.

Various models have been put forward to explain the plagiogranite petrogenesis (see Koepke et al. 2007 for review), including (1) late-stage differentiation of parental MORB-type tholeiitic magma, (2) hydrous partial melting of gabbros or basalts in a MOR setting, (3) silicate immiscibility of an evolved tholeiitic MORB liquid, and (4) assimilation and partial melting of altered dykes below fast spreading ridges.
The latter process for the origin of the studied plagiogranite is excluded by the field data. The contribution of liquid immiscibility to the plagiogranite petrogenesis is conspicuous by the lack of associated strongly $\mathrm{Fe}-\mathrm{Ti}$-enriched mafic rocks. Immiscible $\mathrm{Fe}-\mathrm{Ti}$-enriched mafic rocks associated with plagiogranites are known from the ophiolite suites (Shastry et al. 2001) and experimental immiscible liquids (Dixon \& Rutherford, 1979). Both natural and experimental examples, however, have high $\mathrm{Fe}_{2} \mathrm{O}_{3^{*}}(=24$ $30 \mathrm{wt} \%)$ and $\mathrm{TiO}_{2}(=4.6-8 \mathrm{wt} \%)$ concentrations well above the values in the dykes (Table 2).

Field data do not provide evidence of direct relationships between the gabbro and plagiogranite to constrain the plagiogranite origin by partial melting of the gabbro. The gabbro could be responsible for the plagiogranite origin, based on both occurrences below the lavas in the igneous stratigraphy of the Evros ophiolites (Fig. 2b). The similarity in trace elements and REE patterns and relationships between the plagiogranite and intruding dykes suggest that these dykes are more suitable proxies as parental melts for the plagiogranite origin. To test whether the tholeiitic melts producing dykes that intrude the plagiogranite can account for its composition and petrogenesis, we modelled REE concentrations of the dyke sample Dd2 (modal 0.55 Pl-0.35 Cpx-0.2 Mt). We also evaluated the gabbro sample AG2 (modal 0.50 Pl-0.25 Cpx$0.25 \mathrm{Hbl}$ ) as parent melt in terms of partial melting. The geochemical modelling was carried out assuming Rayleigh fractional crystallization and batch melting. Model REE patterns are compared with those of plagiogranite samples in a chondrite-normalized plot (Fig. 9b). As shown in this diagram, the partial melting of the gabbro could not produce the observed REE patterns of the plagiogranite. Partial melting of the parent material analogous to the tholeiitic dyke for a reasonable degree of 10-20\% melting failed to reproduce LREE patterns of the plagiogranite, whereas fractional crystallization up to $75 \%$ had far greater success in reproducing the REE pattern by overlapping the plagiogranite envelope. These results indicate a simplified fractionation model for the plagiogranite origin via extreme fractionation of the tholeiitic liquid producing the cross-cutting dykes. They provide further evidence for the extent of fractionation (70-85\%) by which plagiogranites could form, as this process has been documented in other Tethyan ophiolites (Floyd, Yaliniz \& Göncüoğlu, 1998; Dilek \& Thy, 2006).

\section{8.b. Comparison with the mafic rocks of extrusive section}

A comparison of the gabbro and dykes in the AgrianiDidymotycho area, with the tholeiitic to boninitic affinity lavas of the extrusive sequence and a boninitic dyke in the Mandrica area, defines significantly overlapping REE and trace element patterns (Figs 6, 7). Noteworthy for these ophiolitic suites are similar trace element ratios (e.g. V/Ti, Th/Ta, Zr/Y; Figs 8a, b, 9a). These features imply genetic relationships between the 
intrusive and extrusive members of the ophiolite suite in the above occurrences, and provide further evidence for a common parentage of the magmatic assemblage formed in a subduction-zone tectonic setting. This comparison clearly points to geochemical continuity of intrusive and extrusive magmatic suites in the tectonomagmatic section of the Evros ophiolites.

\section{8.c. Tectonic significance}

Boninites and arc tholeiites known from the fore-arcs of modern arc/back-arc systems have been related to the early stages of arc systems evolution (Crawford, Falloon \& Green, 1989; Pearce et al. 1992; Stern \& Bloomer, 1992; Bloomer et al. 1995; Taylor, 1995), where they are associated with the plagiogranites (Jafri, Charan \& Govil, 1995). The above magmatic assemblage is documented also in ancient arc systems and ophiolite suites (Coleman \& Donato, 1979; Pearce, Harris \& Tindle, 1984; Pedersen \& Malpas, 1984; Floyd, Yaliniz \& Göncüoğlu, 1998; Luchitskaya, Morozov \& Palandzhyan, 2005), including ophiolites in the Aegean region (Hatzipanagiotou \& Tsikouras, 1999).

Petrological and geochemical data give clear evidence that the gabbro, the plagiogranite and intruding dykes represent tholeiitic-boninitic affinity magmatic products that formed in a supra-subduction zone setting. Chemistry of the studied rocks may well account for locally distinct areas in the source region, implying across-strike variations of the mantle wedge composition. This compositional heterogeneity was induced by subduction zone processes and related input of mantle and slab-derived components that substantially modified the lithospheric material transferred to the overlying mantle wedge. Differentiation processes additionally have contributed to the compositional diversity of the studied magmatic assemblage.

In the case of the studied rocks, the supra-subduction zone assemblage includes tholeiitic-transitional boninitic gabbro, tholeiitic and boninitic dykes and late-stage fractionated melts represented by the plagiogranite. The plagiogranite differs from those associated with mature arcs, and plagiogranite samples fall in the hypothetical ORG field (Fig. 8c), which covers settings in mid-oceanic ridge, fore-arc and back-arc spreading centres. However, the plagiogranite chemistry demonstrates supra-subduction zone affinity similar to that of associated dykes (Figs 6,7). These dykes systematically fill tensile fractures or faults, which suggests the existence of fractured oceanic lithosphere under an extensional regime. The extensional tectonism, as recorded by structures created by the dykes, is clearly associated with the influx of depleted tholeiiticboninitic melts into the fractured brittle oceanic crust. Extension-induced rifting associated with tholeiitic and boninitic-type magmatism has been documented in the fore-arcs of the western Pacific arc systems (Natland \& Tarney, 1981; Pearce et al. 1992; Stern \& Bloomer, 1992; Taylor \& Nesbitt, 1994; Wessel et al.
1994). Therefore, based on chemical affinity and field evidence, we relate the plagiogranite generation to a subduction zone trench-proximal rift-related centre in a fore-arc setting.

Field data and the geochemical signature of the studied magmatic assemblage calls for intrusive products formed in the fore-arc of a supra-subduction zone environment. Because of the geochemical continuity between the intrusive-extrusive suite and equivocal evidence for the association of these magmatic products with a mature arc system, we consider that they mostly represent proto-arc oceanic crust of a nascent arc system. A petro-tectonic scenario for the proto-arc (fore-arc) tholeiitic/boninitic-type magma genesis is discussed below (Fig. 10). We interpret the Agriani gabbro as being a high-level gabbro in the ophiolite suite forming new oceanic crust at the inception of the subduction setting, well before construction of the arc system. In this case, the gabbro forms the oceanic basement of the incipient arc system (Fig. 10a). The sinking cold oceanic crust was overridden by young hot asthenosphere material, providing a thermal gradient at a shallow depth that facilitated melting; the upward migration of the melt exerted extensional stresses in the overlying slab. The sinking graded to subduction and melt extraction following the gabbro segregation has generated melts of the boninitic dykes. The gabbro segregation was accompanied or followed by eruptions as indicated by overlying lavas, which possibly originated in submerged volcanic centres along propagating fracture zones. As subduction progressed under the extensional regime, probably enhanced by slab retreat, further melting in the mantle wedge generated a melt batch of tholeiitic magma. The differentiation of the latter resulted in solidification at a high level to produce the plagiogranite, whose emplacement was succeeded by tholeiitic-boninitic dykes from the deepest part of the melting column (Fig. 10b). As these dykes have enriched the geochemical signature, this suggests replenishment of the mantle wedge by more fertile material, and further melting of that material in association with increasing slab fluxes. Some of the dykes probably acted as feeders for the overlying geochemically similar lavas, which have also boninitic affinity at Didymotycho (Magganas, 2002). This implies that lava eruptions persisted in volcanic centres during and outlasting the plagiogranite intrusion; the extrusive products may provide evidence for compositional continuity towards the mature stage in the eruptive history of the arc system.

In this scenario, the magmatic assemblage records an early stage proto-arc (fore-arc) magma genesis in the tectono-magmatic evolution of the supra-subduction type Evros ophiolites. The northwesterly occurrence (Mandrica area) in the arc-trench system of boninitic dykes intruding sediments at the base of extrusive section, together with continental margin detrital clastics that have been incorporated in the subductionaccretionary assemblage, fits the proto-arc/fore-arc environment. Subsequent evolution of the subduction 

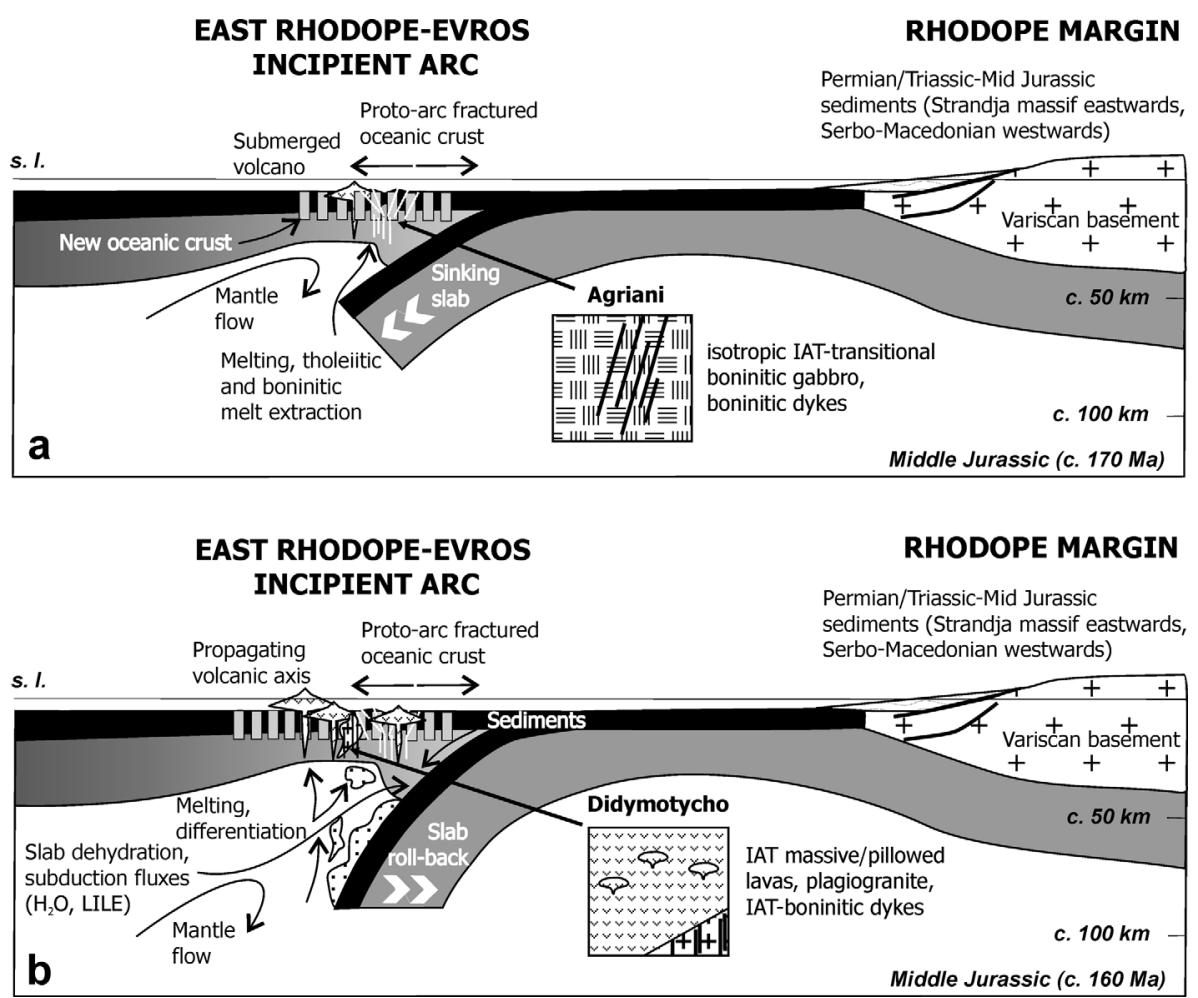

Figure 10. Petro-tectonic scenario for proto-arc/fore-arc oceanic crust generation in the early-stage evolution of supra-subduction-type Evros ophiolites. The position of the gabbroic and plagiogranite bodies and associated dykes in the tectono-magmatic column is indicated. See text for explanation and discussion.

setting towards the mature stage of the arc system occurred afterwards, with the eruption of evolved tholeiitic and calc-alkaline lavas of the extrusive section (Magganas, 2002; Bonev \& Stampfli, 2008).

The Evros ophiolites and counterparts in Bulgaria have been related to intra-oceanic subduction of the Triassic-Jurassic Meliata-Maliac Ocean under the supra-subduction back-arc Vardar Ocean/arc system (Bonev \& Stampfli, 2003). The Vardar Ocean, which by Late Jurassic times had replaced the MeliataMaliac Ocean, developed several arc systems at that time. The Early-Middle Jurassic $(<170 \mathrm{Ma})$ Evros ophiolites represent early supra-subduction zone ophiolites related to the inception of the intraoceanic subduction setting. The Evros ophiolites, together with the Late Jurassic Samothraki Island backarc ophiolites $(155 \pm 7 \mathrm{Ma}$ : Tsikouras, Pe-Piper \& Hatzipanagiotou, 1990; Tsikouras \& Hatzipanagiotou, 1998) immediately south, were likely coupled in an arc/back-arc system (Bonev \& Stampfli, 2008). The propagating Vardarian arc systems, also including a Late Jurassic Paikon arc, Guevgueli (155-164 Ma: Anders et al. 2005) and Chalkidiki ophiolites (see Smith, 1993 for review) of the Vardar zone, have dominated Jurassic-Early Cretaceous geodynamics at the Serbomacedonian-Rhodope margin. Although relations between the above arc systems are hidden in the Aegean Sea, the Evros ophiolites belong to the innermost of them relative to this margin and youngest of all these arcs that were rooted in the Vardar zone of which the Circum-Rhodope Belt (Fig. 1, inset) forms the easternmost part, a feature that allows tracing the Evros ophiolites extension to the Vardar zone, based on the age of ophiolites and character of arc magmatism in the latter, and the subduction systems life cycle ( $c$. 15-20 Ma) in general.

\section{Conclusions}

The field data, petrological and geochemical results from the gabbro, plagiogranite and associated mafic dykes allow us to draw the following conclusions on their petrogenesis and tectonic setting within the tectono-magmatic section of the Evros ophiolites.

(1) Both the Agriani high-level isotropic gabbro and Didymotycho plagiogranite intruded by mafic dykes reveal more extensive occurrences of the plutonic suite underlying the widespread extrusive sequence in the tectono-magmatic section of the Evros ophiolites. These magmatic suites, together with the occurrences of cumulitic rocks and scarce mantle peridotites in the ophiolites, make the Evros ophiolites important Tethyan ophiolites in the north Aegean region, exposing a mantle to crustal ophiolite section.

(2) The low-Ti and high-Ca tholeiitic-transitional boninitic affinity gabbro and intruding boninitic dykes present arc-related petrological and geochemical characteristics with REE and HFS depletion relative to N-MORB, indicative of mantle melts derived from an already depleted 
mantle source in a subduction zone setting. The gabbro most likely originated from melting of a hydrated refractory mantle peridotite overlying the subduction zone. The gabbro segregation succeeded by melts producing the boninitic dykes, which collectively attests to the generation of a new tholeiitic-boninitic oceanic crust. Both magmatic products account for sequential melt extraction in the mantle wedge of the subduction zone.

(3) The plagiogranite, which is classified as a highsilica trondjemite with typical oceanic floor chemistry, is invaded by Fe-rich and low-Ca tholeiitictransitional boninitic dykes. These dykes present subduction zone affinity, with less REE and HFSE depleted geochemical character relative to $\mathrm{N}$ MORB and compared to the gabbro and associated mafic dykes, displaying evolved compositions and a differentiated trend. The origin of plagiogranite via extreme fractional crystallization of the tholeiitic liquid from which intruding dykes had crystallized is indicated by REE modelling. The distinct chemistry of the dykes intruding the plagiogranite suggests crystallization from a magma batch likely reflecting replenishment of the mantle wedge by more fertile asthenospheric material that has been substantially modified by subduction zone components.

(4) As the mafic dykes systematically fill tensile fractures or faults, this indicates their emplacement in an extensional tectonic regime. This extensional regime is coherent on a sub-regional scale as recorded by a consistent NE-SW trend of the mafic dykes in both the gabbroic and plagiogranite bodies. The extension-dominated setting, as well as a strong arc signature and geochemical affinity of the magmatic assemblage, are all features which call for trench-proximal fore-arc tholeiitic and boninitic-type magma genesis. The studied intrusive rock suite is therefore interpreted as a magmatic assemblage that tracks the early stages of proto-arc/fore-arc oceanic crust generation in the supra-subduction zone-type Evros ophiolites.

(5) A comparison of the studied mafic intrusive rocks with the mafic lavas of the extrusive sequence showed significantly overlapping REE and trace element patterns, implying geochemical continuity and genetic relationships between the intrusive and extrusive suites of the Evros ophiolites, thus providing further evidence for a common parentage of plutonic members and their extrusive equivalents in the supra-subduction zone magmatic assemblage.

Acknowledgements. The post-doctoral research grant from AUF (NB) is gratefully acknowledged. Field work in Greece was supported by the University of Lausanne. Reviews by Y. Dilek and A. Magganas and editorial comments by D.
Pyle helped to improve the paper. Supplementary appendix is available at http://www.cambridge.org/journals/geo.

\section{References}

ALDISS, D. T. 1981. Plagiogranites from the ocean crust and ophiolites. Nature 289, 577-8.

Anders, B., Reischmann, T., Poller, U. \& Kostopoulos, D. 2005. Age and origin of granitic rocks of the eastern Vardar Zone, Greece: new constraints on the evolution of the Internal Hellenides. Journal of the Geological Society, London 162, 857-70.

ANDERSON, J. L. \& SMITH, D. R. 1995. The effects of temperature and $f \mathrm{O}_{2}$ on the Al-in-hornblende barometer. American Mineralogist 80, 549-59.

ARCulus, R. J. \& WILLS, K. J. A. 1980. The petrology of plutonic blocks and inclusions from Lesser Antilles island arc. Journal of Petrology 21, 743-99.

BARKER, F. \& ARTH, J. G. 1976. Generation of trondhjemitictonalitic liquids and Archean bimodal trondhjemitebasalt suites. Geology 4, 596-600.

BEARD, J. S. 1986. Characteristic mineralogy of arc-related cumulate gabbros: implications for the tectonic setting of gabbroic plutons and for andesite genesis. Geology 14, 848-51.

Beccaluva, L., Coltorti, M., Giunta, G. \& Siena, F. 2004. Tethyan v. Cordilleran ophiolites: reappraisal of distinctive tectono-magmatic features of suprasubduction complexes in relation to the subduction mode. Tectonophysics 393, 163-74.

Beccaluva, L., Macciotta, G., Piccardo, G. B. \& ZEDA, O. 1989. Clinopyroxene compositions of ophiolitic basalts as petrogenetic indicator. Chemical Geo$\log y 77,165-82$.

Biggazzi, G., Del Moro, A., InNocenti, F., KyriakoPOUlOS, K., MANETTI, P., PAPAdOPOUlOS, P., Norelliti, P. \& MAgGanAS, A. 1989. The magmatic intrusive complex of Petrota, west Thrace: age and geodynamic significance. Geologica Rhodopica 1, 290-7.

BloOmer, S. H., TAYlor, B., MACLEOD, C. J., Stern, R. J., FrYER, P., HAWKINS, J. W. \& JOHNSON, L. 1995. Early arc volcanism and the ophiolite problem: a perspective from drilling in the western Pacific. In Active Margins and Marginal Basins of the Western Pacific (eds B. Taylor \& J. Natland), pp. 1-30. AGU Geophysical Monograph no. 88, Washington, D.C.

BonEV, N. 2005. Foraminifers from the exotic Late Permian limestone pebbles in the Mesozoic low-grade sequence of the eastern Rhodope, Bulgaria: paleogeographic and paleotectonic consequences. Neues Jahrbuch für Geologie und Paläontologie Monatschefte 7, 385-403.

BonEV, N. 2006. Cenozoic tectonic evolution of the eastern Rhodope Massif (Bulgaria): Basement structure and kinematics of syn- to postcollisional extensional deformation. In Postcollisional Tectonics and Magmatism in the Mediterranean region and Asia (eds Y. Dilek \& S. Pavlides), pp. 211-35. Geological Society of America, Special Paper no. 409.

Bonev, N., Burg, J.-P. \& IVANov, Z. 2006. Structural evolution of an extensional gneiss dome - the KesebirKardamos dome, E. Rhodope, Bulgaria-Greece. International Journal of Earth Sciences 95, 318-40.

Bonev, N., MARcheV, P. \& Singer, B. 2006. ${ }^{40} \mathrm{Ar} /{ }^{39} \mathrm{Ar}$ geochronology constraints on the Middle Tertiary basement extensional exhumation, and its relation to ore-forming and magmatic processes in the eastern Rhodope (Bulgaria). Geodinamica Acta 19, 267-82. 
Bonev, N. G. \& Stampfli, G. M. 2003. New structural and petrologic data on Mesozoic schists in the Rhodope (Bulgaria): geodynamic implications. Comptes Rendus Geoscience 335, 691-9.

BONEV, N. \& STAMPFLI, G. 2005. Compositional diversity of the Evros ophiolite, Thrace, northeastern Greece: field occurrences, preliminary petrologic and geochemical data on plutonic sequence and tectonic implications. Proceedings of the Jubilee International Conference 80th Year Bulgarian Geological Society (eds Y. Yanev \& R. Nedialkov), pp. 31-4. Sofia University Press.

Bonev, N. \& Stampfli, G. 2008. Petrology, geochemistry and tectonic implications of Jurassic island arc magmatism as revealed by mafic volcanic rocks in the Mesozoic low-grade sequence of the eastern Rhodope, Bulgaria. Lithos 100, 210-33.

Bortolotti, V., Marroni, M., Pandolfi, L., PrinciPi, G. \& SACCANI, E. 2002. Interaction between mid-ocean ridge and subduction magmatism in Albanian ophiolites. Journal of Geology 110, 561-76.

BortolotTI, V. \& PRINCIPI, G. 2005. Tethyan ophiolites and Pangea break-up. The Island Arc 14, 442-70.

BOYANOV, I. \& BODUROV, K. 1979. Triassic conodonts in carbonate breccia within the low-grade metamorphic rocks of the East Rhodopes. Geologica Balcanica 9, 97-104.

Boyanov, I. \& Goranov, A. 2001. Late Alpine (Palaeogene) superimposed depressions in parts of Southeast Bulgaria. Geologica Balcanica 31, 3-36.

BOYANOV, I. \& LiPMAN, P. 1973. On the Lower Cretaceous age of the low-crystalline metamorphic complex in the East Rhodopes. Comptes Rendus de l'Académie bulgare des Sciences 26, 1225-6.

BOYANOV, I. \& RUSSEVA, M. 1989. Lithostratigraphy and tectonic position of the Mesozoic rocks from the East Rhodopes. Geologica Rhodopica 1, 22-33.

BoyAnOV, I., RusSEVA, K. M. \& Dimitrova, E. 1982. First find of Upper Cretaceous foraminifers in East Rhodopes. Geologica Balcanica 4, 20.

BoyAnOV, I., Russeva, M., Toprakcieva, V. \& Dimitrova, E. 1990. Lithostratigraphy of the Mesozoic rocks from the Eastern Rhodopes. Geologica Balcanica 20, 3-28.

BuRG, J.-P., RicoU, L.-E., IVANOV, Z., DimOV, D. \& KLAIN, L. 1996. Crustal-scale thrust complex in the Rhodope Massif. Structures and kinematics. Terra Nova 8, $6-15$.

Cameron, W. E., Nisbet, E. G. \& Dietrich, V. J. 1979. Boninites, komatiites and ophiolitic basalts. Nature $\mathbf{2 8 0}$, 550-3.

Carrigan, C., Mukasa, S., Haydoutov, I. \& Kolcheva, K. 2003. Ion microprobe U-Pb zircon ages of pre-Alpine rocks in the Balkan, Sredna Gora and Rhodope terranes of Bulgaria: constraints on Neoproterozoic and Variscan evolution. Journal of Czech Geological Society 48 , 32-3.

Coleman, R. G. \& Donato, M. M. 1979. Oceanic plagiogranite revisited. In Trondhjemites, dacites, and related rocks (ed. F. Barker), pp. 149-68. Amsterdam: Elsevier.

Coleman, R. G. \& Peterman, Z. E. 1975. Oceanic plagiogrante. Journal of Geophysical Research 80, $1099-1108$

Crawford, A. J., Falloon, T. J. \& Green, D. H. 1989. Classification, petrogenesis and tectonic setting of boninites. In Boninites and Related Rocks (ed. A. J. Crawford), pp. 1-49. London: Unwin Hyman.

DE BARI, S. M. \& COLEMAN, R. G. 1989. Examination of the deep levels of an island arc: evidence from the Tonisia ultramafic-mafic assemblage, Tonisia, Alaska. Journal of Geophysical Research 94, 4373-91.

Del Moro, A., InNocenti, F., Kyriakopoulos, C., MANETTI, P. \& PAPAdOPOUlOS, P. 1988. Tertiary granitoids from Thrace (northern Greece): Sr isotopic and petrochemical data. Neues Jahrbuch für Geologie und Paläontologie Abhandlungen 159, 113-35.

Dilek, Y., Furnes, H. \& Shallo, M. 2007. Suprasubduction zone ophiolite formation along the periphery of Mesozoic Gondwana. Gondwana Research 11, 453-75.

DileK, Y. \& THY, P. 2006. Age and petrogenesis of plagiogranite intrusions in the Ankara mélange, central Turkey. Island Arc 15, 44-57.

DimADIS, L. \& NiKOLOV, T. 1997. An ammonite find in the Makri unit (Berriasian, southeast Rhodopes, northeast Greece). Comptes Rendus de l'Académie bulgare des Sciences 50, 71-4.

Dimadis, L., PAPAdopolous, P., Goranov, A. \& ENCHEVA, M. 1996. First biostratigraphic evidence for the presence of Triassic at Melia (Western Thrace, Greece). Geologica Balcanica 26, 37-40.

DINTER, D. A. 1998. Late Cenozoic extension of the Alpine collisional orogen, northeastern Greece: Origin of the north Aegean basin. Geological Society of America Bulletin 110, 1208-30.

DinTER, D. A., MACFARlANE, A. M., HAMES, W., ISACHSEN, C., BOWRING, S. \& RoYden, L. 1995. U-Pb and ${ }^{40} \mathrm{Ar} /{ }^{39} \mathrm{Ar}$ geochronology of the Symvolon granodiorite: implications for the thermal and structural evolution of the Rhodope metamorphic core complex, northeastern Greece. Tectonics 14, 886-908.

DiXON, S. \& RUTHERFORD, M. J. 1979. Plagiogranites as late-stage immiscible liquids in ophiolite and mid-ocean ridge suites: an experimental study. Earth and Planetary Science Letters 45, 45-60.

Dupuy, C., Dostal, J., Mercelot, G., Bougault, H., JORON, J. L. \& TREUIL, M. 1982. Geochemistry of basalts from central and southern New Hebrides arcs: implication for their source rock composition. Earth and Planetary Science Letters 60, 207-25.

Elliott, T., Plank, T., ZindleR, A., White, W. \& BOURDON, B. 1997. Element transport from slab to volcanic front at Mariana arc. Journal of Geophysical Research 102, 14991-15019.

FloYd, P. A., YAlinIZ, M. K. \& GÖNCÜOĞLU, M. C. 1998. Geochemistry and petrogenesis of intrusive and extrusive ophiolitic plagiogranites, Central Anatolian Crystalline Complex, Turkey. Lithos 42, 225-41.

Frass, A., Hegewald, S., Kloos, R. M., Tesch, C. \& ARIKAS, K. 1990. The geology of the graben of Petrota (Thrace, NE Greece). Geologica Rhodopica 2, 50-63.

HARKovSKA, A., YANEV, Y. \& MARCHEV, P. 1989. General features of the Paleogene orogenic magmatism in Bulgaria. Geologica Balcanica 19, 37-72.

HATZIPANAGIOTOU, K. \& TSIKOURAS, B. 1999. Plagiogranites in the Hellenic ophiolites. Ofioliti 24, 283-92.

Haydoutov, I., KolcheVA, K., DAIEVA, L.-A., SAVOV, I. \& CARRIGAN, C. 2004. Island-arc origin of the variegated formations from the East Rhodope, Bulgaria - implications for the evolution of the Rhodope Massif. Ofioliti 29, 145-57.

HÉBERT, R. \& LAURENT, R. 1990. Mineral chemistry of the plutonic section of the Troodos ophiolite: new constraints for genesis of arc-related ophiolites. In Proceedings of Troodos Ophiolite Symposium (eds J. Malpas, E. Moores, A. Panayotou \& C. Xenophontos), pp. 149-63. Geological Survey of Cyprus. 
Hole, M. J., SAunders, A. D., Marriner, G. F. \& TARney, J. 1984. Subduction of pelagic sediments: implications for the origin of Ce-anomalous basalts from the Mariana Islands. Journal of the Geological Society, London 141, 453-72.

Innocenti, F., Kolios, N., Manetti, P., Mazzuli, R., PeCCERILlo, A., RitA, F. \& Villari, L. 1984. Evolution and geodynamic significance of Tertiary orogenic volcanism in northeastern Greece. Bulletin of Volcanology $47,25-37$.

Irvine, T. H. \& Baragar, W. R. A. 1971. A guide to the chemical classification of the common volcanic rocks. Canadian Journal of Earth Sciences 8, 523-48.

IVANOV, R. \& KoPP, K. O. 1969. Das Alttertiär Thrakiens und der Ostrhodope. Geologica et Paleontologica 3, 123-51.

JAFri, S. H., Charan, S. N. \& GoviL, P. K. 1995. Plagiogranite from the Andaman ophiolite belt, Bay of Bengal, India. Journal of the Geological Society, London 154, 681-8.

JARANOV, D. 1960. Tectonics of Bulgaria. Sofia: Technica, 283 pp. (in Bulgarian).

KauffmanN, G., Kockel, F. \& Mollat, H. 1976. Notes on the stratigraphic and paleogeographic position of the Svoula formation in the Innermost Zone of the Hellenides (Northern Greece). Bulletin de la Societe Géologique de France 18, 225-30.

Kockel, F., Mollat, H. \& Walther, H. W. 1977. Erlauterungen zur geologicschen Karte der Chalkidiki und angrenzender Gebiete 1/100.000 (Nord Griechenland). Hanover: Bundesanstalt für Geowissenschaften und Rohstoffe, $119 \mathrm{pp}$.

Koepke, J., Berndt, J., Feig, S. T. \& Holtz, F. 2007. The formation of $\mathrm{SiO}_{2}$-rich melts within the deep oceanic crust by hydrous partial melting of gabbros. Contributions to Mineralogy and Petrology 153, 67-84.

Koglin, N., ReISCHMANN, T., Kostopoulos, D., MATUKOV, D. \& SERgEeV, S. 2007. Zircon SHRIMP ages and the origin of ophiolitic rocks from the NE Aegean region, Greece. Geophysical Research Abstracts 9, paper 06848.

KopP, K. O. 1969. Geologie Thrakiens VI: Der Coban Dag westlich von Alexandroupolis. Geotektonische Forschungen 31, 97-116.

KroHe, A. \& Mroskos, E. 2002. Multiple generations of extensional detachments in the Rhodope Mountains (northern Greece): evidence of episodic exhumation of high-pressure rocks. In The Timing and Location of Major Ore Deposits in an Evolving Orogen (eds D. J. Blundell, F. Neubauer \& A. von Quadt), pp. 151-78. Geological Society of London, Special Publication no. 204.

LE MAITRE, R. W. 2002. Igneous Rocks: a Classification and Glossary of Terms. Recommendations of the International Union of Geological Sciences, Subcommission on the Systematics of Igneous Rocks, 2nd edition. Cambridge: Cambridge University Press.

Leterrier, J., Maury, R. C., Thonon, P., Girard, D. \& MARCHAL, M. 1982. Clinopyroxene composition as a method of identification of the magmatic affinities of paleo-volcanic series. Earth and Planetary Science Letters 59, 139-54.

LIATI, A. 2005. Identification of repeated Alpine (ultra) high-pressure metamorphic events by U-Pb SHRIMP geochronology and REE geochemistry of zircon: the Rhodope zone of Northern Greece. Contributions to Mineralogy and Petrology 150, 608-30.

LindSley, D. H. 1983. Pyroxene thermometry. American Mineralogist 68, 477-93.
Lips, A. L. W., White, S. H. \& WiJbrans, J. R. 2000. Middle-Late Alpine thermotectonic evolution of the southern Rhodope Massif, Greece. Geodinamica Acta 13, 281-92.

Luchitskaya, M. V., Morozov, O. L. \& Palandzhyan, S. A. 2005. Plagiogranite magmatism in the Masozoic island-arc structure of the Pekulney Ridge, Chukotka Peninsula, NE Russia. Lithos 79, 251-69.

MagGanAS, A. 2007. Plagiogranitic rocks of Evros ophiolite, NE Greece. Bulletin of the Geological Society of Greece 40, 884-98.

MagGanAS, A. C. 2002. Constraints on the petrogenesis of Evros ophiolite extrusives, NE Greece. Lithos 65, 16582.

Magganas, A., Sideris, C. \& KoKkinaKis, A. 1991. Marginal basin-volcanic arc origin of metabasic rocks of the Circum-Rhodope Belt, Thrace, Greece. Mineralogy and Petrology 44, 235-52.

MARATOS, G. \& ANDRONOPOULOS, B. 1964. Nouvelles données sur l'age des phyllites du Rhodope. Bulletin of the Geological Society of Greece 6, 113-32.

McCulloch, M. T. \& GAMBle, J. A. 1991. Geochemical and geodynamical constraints on subduction zone magmatism. Earth and Planetary Science Letters 102, 358-74.

Mukasa, S., Haydoutov, I., CARrigan, C. \& Kolcheva, K. 2003. Thermobarometry and ${ }^{40} \mathrm{Ar} /{ }^{39} \mathrm{Ar}$ ages of eclogitic and gneissic rocks in the Sredna Gora and Rhodope terranes of Bulgaria. Journal of Czech Geological Society 48, 94-5.

Murton, B. J., Peate, D. W., Arculus, R. J., Pearce, J. A. \& VAN DER LAAN, S. 1992. Trace-element geochemistry of volcanic rocks from Site 786: the Izu-Bonin forearc. In Proceedings of the Ocean Drilling Program, Scientific Results, vol. 125 (eds P. Fryer, V. L. Pearce, L. Stokking et al.), pp. 211-35. College Station, Texas.

NATLAND, J. H. \& TARNEY, J. 1981. Petrologic evolution of the Mariana arc and back-arc system - a synthesis of drilling results in the South Philippine Sea. Initial Reports of Deep Sea Drilling Program 60, 877-908. College Station, Texas.

PAMic, J., TOMLJENOVIĆ, B. \& BALEN, D. 2002. Geodynamic and petrogenetic evolution of Alpine ophiolites from the central and NW Dinarides: an overview. Lithos 65, 11342.

PANJASAWATWONG, Y., DANYUSHEVSKY, L. V., CRAWFORD, A. J. \& HARRIS, K. L. 1997. An experimental study of the effects of melt composition on plagioclase-melt equilibrium at 5 and 10 kbars: implications for the origin of magmatic high-An plagioclase. Contributions to Mineralogy and Petrology 118, 420-32.

PAPADOPOUlOS, P. \& ANASTASIADIS, I. 2002. Geological map of Greece, scale 1:200 000 sheet Rhodopi-Thrace. Xanthi: IGME.

PAPAdopoulos, P., ARVANitidis, N. \& Zanas, I. 1989. Some preliminary geological aspects on the Makri unit (Phyllite series), Peri-Rhodope Zone. Geologica Rhodopica 1, 34-42.

PAPANIKOLAOU, D. 1997. The tectonostratigraphic terranes of the Hellenides. Annales géologiques des Pays Hellénique 37, 495-514.

PEARCE, J. A. 1996. A user's guide to basalt discrimination diagrams. In Trace Element Geochemistry of Volcanic Rocks: Application to massive sulphide exploration (ed. D. A. Wyman), pp. 79-113. Geological Association of Canada, Short Course Notes no. 12.

Pearce, J. A., Harris, N. B. W. \& Tindle, A. G. 1984. Trace element discrimination diagrams for the tectonic 
interpretation of granitic rocks. Journal of Petrology 25, 956-83.

Pearce, J. A., Kempton, P. D., Nowell, G. M. \& NoBlE, S. R. 1999. Hf-Nd element and isotope perspective on the nature and provenance of mantle and subduction component in western Pacific arc-basin systems. Journal of Petrology 40, 1597-1611.

Pearce, J. A., LipPard, S. J. \& RoberTs, S. 1984. Characteristics and tectonic significance of supra-subduction zone ophiolites. In Marginal Basin Geology: Volcanic and Associated Sedimentary and Tectonic Processes in Modern and Ancient Marginal Basins (eds B. P. Kokelaar \& M. F. Howells), pp. 77-94. Geological Society of London, Special Publication no. 16.

Pearce, J. A. \& Norry, M. J. 1979. Petrogenetic implications of $\mathrm{Ti}, \mathrm{Zr}, \mathrm{Y}$, and $\mathrm{Nb}$ variations in volcanic rocks. Contributions to Mineralogy and Petrology 69, 33-47.

Pearce, J. A., Van Der LaAn, S. R., Arculus, R. J., Murton, B. J., Ishit, T., Peate, D. W. \& PARkinson, I. J. 1992. Boninite and harzburgite from Leg 125 (BoninMariana forearc): a case study of magma genesis during the initial stages of subduction. In Proceedings of the Ocean Drilling Program, Scientific Results, vol. 125 (eds P. Fryer, V. L. Pearce, L. Stokking et al.), pp. 623-60. College Station, Texas.

Pedersen, R. B. \& Malpas, J. 1984. The origin of oceanic plagiogranites from the Karmoy ophiolite, western Norway. Contributions to Mineralogy and Petrology $\mathbf{8 8}$, $36-52$.

Peytcheva, I., Kostitsin, Y., Salnikova, E., von Quadt, A., KamenOV, B. \& KlaIN, L. 1999. Alpine evolution of the magmatism in the West-Rhodopes: $\mathrm{Rb}-\mathrm{Sr}$ and $\mathrm{U}-\mathrm{Pb}$ isotope data. Journal of Conference Abstracts 4, 470.

PeYtcheVA, I. \& QuADT, A. V. 1995. U-Pb zircon dating of metagranites from Byala Reka region in the east Rhodopes, Bulgaria. In Proceedings of XV Congress of Carpatho-Balkan Geological Association (ed. D. Papanikolaou), pp. 637-42. Geological Society of Greece, Special Publication no. 4.

Ricou, L.-E., Burg, J.-P., GodfriauX, I. \& IVANOV, Z. 1998. The Rhodope and Vardar: the metamorphic and the olistostromic paired belts related to the Cretaceous subduction under Europe. Geodinamica Acta 11, 285309.

Robertson, A. H. F. 2002. Overview of the genesis and emplacement of Mesozoic ophiolites in the Eastern Mediterranean Tethyan region. Lithos 65, 1-67.

Robertson, A. H. F., Dixon, J. E., Brown, S., Collins, A., Morris, A., Pickett, E., Sharp, I. \& UstaÖmer, T. 1996. Alternative tectonic models for the Late Paleozoic-Early Tertiary development of the Tethys in the Eastern Mediterranean region. In Paleomagnetism and Tectonics of the Mediterranean region (eds A. Morris \& D. H. Tarling), pp. 239-63. Geological Society of London, Special Publication no. 105.

Rollinson, H. 1993. Using geochemical data: evaluation, presentation, interpretation. UK: Longman Group, $352 \mathrm{pp}$.

Shastry, A., Srivastava, R. K., Chandra, R. \& GenNER, G. A. 2001. Fe-Ti-enriched mafic rocks from south Andaman ophiolite suite: implication of late stage liquid immiscibility. Current Science 80, 453-4.

SHERVAIS, J. W. 1982. Ti-V plots and the petrogenesis of modern ophiolitic lavas. Earth and Planetary Science Letters 57, 101-18.

Sisson, T. W. \& Grove, T. L. 1993. Experimental investigation of the role of $\mathrm{H}_{2} \mathrm{O}$ in calc-alkaline differentiation and subduction zone magmatism. Contributions to Mineralogy and Petrology 113, 143-66.

SMITH, A. G. 1993. Tectonic significance of Hellenic-Dinaric ophiolites. In Magmatic Processes and Plate Tectonics (eds H. M. Prichard, T. Alabaster, N. B. W. Harris \& C. R. Neary), pp. 213-43. Geological Society of London, Special Publication no. 76.

SoldATOS, T. \& ChRISTOFIDES, G. 1986. Rb-Sr geochronology and origin of the Elatia Pluton, Central Rhodope, North Greece. Geologica Balcanica 16, 15-23.

StAMPFLI, G. M. 2000. Tethyan oceans. In Tectonics and Magmatism in Turkey and Surrounding Region (eds E. Bozkurt, J. A. Winchester \& J. D. A. Piper), pp. 1-23. Geological Society of London, Special Publication no. 173.

StAMPfli, G. M. \& Borel, G. D. 2002. A plate tectonic model for the Paleozoic and Mesozoic costrained by dynamic plate boundaries and restored synthetic oceanic isochrons. Earth and Planetary Science Letters 196, $17-33$.

StAMPfli, G. M. \& Borel, G. D. 2004. The TRANSMED transects in space and time: constraints on the paleotectonic evolution of the Mediterranean domain. In The TRANSMED Atlas: the Mediterranean Region from Crust to Mantle (eds W. Cavazza, F. Roure, W. Spakman, G. M. Stampfli \& P. Ziegler), pp. 53-90. Berlin: Springer Verlag.

Stern, R. J. \& Bloomer, S. H. 1992. Subduction zone infancy: examples from the Eocene Izu-Bonin-Mariana and Jurassic California arcs. Geological Society of America Bulletin 104, 1621-36.

Sun, S. S. \& McDonough, W. F. 1989. Chemical and isotopic systematics of ocean basalts: implications for mantle composition and processes. In Magmatism in Ocean Basins (eds A. D. Saunders \& M. J. Norry), pp. 313-45. Geological Society of London, Special Publication no. 42

Tikhomirova, L. B., Boyanov, I. \& ZagorCheV, I. 1988. Early Jurassic radiolarians from the Eastern Rhodopes: a revision of the age of Dolno Lukovo Formation. Geologica Balcanica 18, 58.

TRIFOnOVA, E. \& BoyAnOV, I. 1986. Late Permian foraminifers from rock fragments in the Mesozoic phyllitoid formation of the East Rhodopes, Bulgaria. Geologica Balcanica 16, 25-30.

TRIKKALINOS, J. K. 1955. Beiträge zur Erforschung des tektonischen Baues Griechenlands. Über das Alter der vortertiaren Schichten des Gebietes von AlexandroupolisDidymotichon-Westthrazien. Annales Géologiques des Pays Hellénique 1, 81-2.

Taylor B. (ed.) 1995. Backarc basins: tectonics and magmatism. New York: Plenum Press, 524 pp.

TAYLOR, R. N. \& NeSBITT, R. W. 1994. Arc volcanism in an extensional regime at the initiation of subduction a geochemical study of Hahajima, Bonin Islands, Japan. In Volcanism Associated with Extension at Consuming Plate Margins (ed. J. L. Smellie), pp. 115 34. Geological Society of London, Special Publication no. 81 .

Tsikouras, B. \& Hatzipanagiotou, K. 1998. Petrogenetic evolution of an ophiolite fragment in an ensialic marginal basin, northern Aegean (Samothraki Island, Greece). European Journal of Mineralogy 10, 551-67.

Tsikouras, B., Pe-Piper, G. \& HatziPAnagiotou, K. 1990. A new date for an ophiolite on the northeastern margin of the Vardar zone, Samothraki, Greece. Neues Jahrbuch für Mineralogie Monatschefte 11, 521-7. 
VON BRAUN, E. 1968. Die mesozoischen Hüllgesteine der SE-Rhodopen in Westthrazien (Griechenland). Geologisches Jahrbuch 85, 565-84.

Wessel, J. K., Fryer, P., Wessel, P. \& TAYlor, B. 1994. Extension in the Northern Mariana Inner Forearc. Journal of Geophysical Research 99, 15181-203.

Winchester, J. A. \& Floyd, P. A. 1977. Geochemical discrimination of different magma series and their differentiation products using immobile elements. Chemical Geology 20, 325-43.
WooD, D. A. 1980. The application of a Th-Hf-Ta diagram to problems of tectonomagmatic classification and to establishing the nature of crustal contamination of basaltic lavas of the British Tertiary volcanic province. Earth and Planetary Science Letters 50, $11-30$.

WoOdHEAD, J. D., EgGins, S. M. \& Johnson, R. W. 1998. Magma genesis in the New Britain island arc: Further insights into melting and mass transfer processes. Journal of Petrology 39, 1641-8. 